GSI-Preprint-2000-49, November 2000

\title{
Shadowing Effects on Vector Boson Production
}

\author{
R. Vogt \\ GSI, Planckstrasse 1, D-64291 Darmstadt \\ Nuclear Science Division, Lawrence Berkeley National Laboratory, Berkeley, CA 94720, USA \\ Physics Department, University of California, Davis, CA 95616, USA
}

\begin{abstract}
We explore how nuclear modifications to the nucleon structure functions, shadowing, affect massive gauge boson production in heavy ion collisions at different impact parameters. We calculate the dependence of $Z^{0}, W^{+}$and $W^{-}$production on rapidity and impact parameter to next-to-leading order in $\mathrm{Pb}+\mathrm{Pb}$ collisions at $5.5 \mathrm{TeV} /$ nucleon to study quark shadowing at high $Q^{2}$. We also compare our $\mathrm{Pb}+\mathrm{Pb}$ results to the $p p$ rapidity distributions at 14 $\mathrm{TeV}$.
\end{abstract}

Typeset using REVTEX 
The heavy ion collisions at the LHC will be a rich testing ground for hard processes which should dominate particle production [1,2]. One of the most promising signatures of quark-gluon plasma production at the CERN SPS is $J / \psi$ suppression [3, 团 which has been compared to the Drell-Yan continuum in the lepton pair mass range $2.9<m<4.5 \mathrm{GeV}$ [5]. Both $J / \psi$ and Drell-Yan pair production are calculable in perturbative QCD. At the LHC, quarkonium suppression will be difficult to compare to the dilepton continuum due to contributions from $c \bar{c}$ and $b \bar{b}$ decays which have large uncertainties in nuclear collisions [6]. Since the low mass dilepton continuum is expected to be dominated by $b \bar{b}$ decays, the $Z^{0}$ was suggested as an alternative reference process for quarkonium suppression at the LHC [7.88]. There are two difficulties with using the $Z^{0}$ as a baseline for quarkonium suppression: the large mass differences, $m_{Z^{0}} \gg m_{\Upsilon}, m_{J / \psi}$, and the difference in production mechanisms, predominantly $q \bar{q}$ for the $Z^{0}$ and $g g$ for quarkonium. Both these differences are important as far as nuclear effects are concerned. However, the differences that reduce the value of the $Z^{0}$ as a baseline process are the same that make it an interesting object of study itself- the $Z^{0}$ provides a unique opportunity to study the modifications of the quark distributions in the nucleus at high $Q^{2}$. Therefore, in this paper we examine the possible effects of this shadowing on $Z^{0}$ production as well as $W^{+}$and $W^{-}$production which are also quark dominated. The impact parameter dependence of the shadowing effect will also be discussed.

We further address the issue of how to measure the shadowing effect. Since isospin will play an important role in quark-dominated processes, the comparison between $\mathrm{Pb}+\mathrm{Pb}$ interactions with and without shadowing is less useful than in gluon-dominated processes such as heavy quark production [9]. In addition, the first, best, $p p$ data will be at the maximum LHC energy of $14 \mathrm{TeV}$. Therefore we will also present the predicted rapidity distributions in $\mathrm{Pb}+\mathrm{Pb}$ collisions at $5.5 \mathrm{TeV} /$ nucleon, the $p p$ distributions at $14 \mathrm{TeV}$, and the $\mathrm{Pb}+\mathrm{Pb} / p p$ ratios as a function of rapidity at the two energies.

The electroweak production and decay channels of the massive vector bosons make them excellent candidates for shadowing studies since no hadronic final-state rescattering is possible. The $Z^{0}$ itself, with a $3.37 \%$ branching ratio to lepton pairs, will be easily observable by reconstructing the peak in the dilepton spectra. Full reconstruction of the leptonic $W^{ \pm}$ decays, $W^{ \pm} \rightarrow l^{ \pm} \nu$, is not possible due to the missing energy given to the undetected neutrino but charged leptons with momenta greater than $40 \mathrm{GeV}$ should be prominent. This decay channel has been used at the Tevatron to measure the asymmetry between $W^{+}$and $W^{-}$production since the asymmetry is sensitive to the down to up quark ratio in the proton at intermediate values of $x$ and high $Q^{2}$ [10]. If the charged leptons from $W^{ \pm}$decays can be identified in heavy ion collisions, such asymmetry measurements may also be employed at the LHC to reduce systematic uncertainties and obtain a more meaningful determination of the $Q^{2}$ dependence of quark shadowing in the nucleus.

The next-to-leading order, NLO, cross section per nucleon for nuclei $A$ and $B$ colliding at impact parameter $b$ and producing a vector boson $V$ with mass $m$ at scale $Q$ is

$$
\begin{aligned}
\frac{1}{A B} \frac{d \sigma_{A B}^{V}}{d y d^{2} b d^{2} r}= & H_{i j}^{V} \int d z d z^{\prime} d x_{1} d x_{2} d x \delta\left(\frac{m_{V}^{2}}{s}-x x_{1} x_{2}\right) \delta\left(y-\frac{1}{2} \ln \left(\frac{x_{1}}{x_{2}}\right)\right) \\
& \times\left\{\sum_{i, j \in Q, \bar{Q}} C^{\mathrm{ii}}\left(q_{i}, \bar{q}_{j}\right) \Delta_{q \bar{q}}(x) F_{q_{i}}^{A}\left(x_{1}, Q^{2}, \vec{r}, z\right) F_{\bar{q}_{j}}^{B}\left(x_{2}, Q^{2}, \vec{b}-\vec{r}, z^{\prime}\right)\right.
\end{aligned}
$$




$$
\begin{aligned}
& +\sum_{i, k \in Q, \bar{Q}} C^{\mathrm{if}}\left(q_{i}, q_{k}\right) \Delta_{q g}(x)\left[F_{q_{i}}^{A}\left(x_{1}, Q^{2}, \vec{r}, z\right) F_{g}^{B}\left(x_{2}, Q^{2}, \vec{b}-\vec{r}, z^{\prime}\right)\right. \\
& \left.\left.+F_{g}^{A}\left(x_{1}, Q^{2}, \vec{r}, z\right) F_{q_{j}}^{B}\left(x_{2}, Q^{2}, \vec{b}-\vec{r}, z^{\prime}\right)\right]\right\}
\end{aligned}
$$

where $H_{i j}^{V}$ is proportional to the leading order, LO, partonic $i j \rightarrow V$ cross section and $Q=u, d, s$ and $c$. The matrices $C^{\mathrm{ii}}$ and $C^{\mathrm{if}}$ contain information on the coupling of the various quark flavors to boson $V$.

We assume that the parton densities $F_{i}^{A}\left(x, Q^{2}, \vec{r}, z\right)$ can be factorized into $x$ and $Q^{2}$ independent nuclear density distributions, position and nuclear-number independent nucleon parton densities, and a shadowing function $S^{i}\left(A, x, Q^{2}, \vec{r}, z\right)$ that describes the modification of the nuclear structure functions in position and momentum space. Thus we have

$$
\begin{aligned}
F_{i}^{A}\left(x, Q^{2}, \vec{r}, z\right) & =\rho_{A}(s) S^{i}\left(A, x, Q^{2}, \vec{r}, z\right) f_{i}^{N}\left(x, Q^{2}\right) \\
F_{j}^{B}\left(x, Q^{2}, \vec{b}-\vec{r}, z^{\prime}\right) & =\rho_{B}\left(s^{\prime}\right) S^{j}\left(B, x, Q^{2}, \vec{b}-\vec{r}, z^{\prime}\right) f_{j}^{N}\left(x, Q^{2}\right)
\end{aligned}
$$

where $f_{i}^{N}\left(x, Q^{2}\right)$ is the density of parton $i$ in the nucleon and the radial variables $s$ and $s^{\prime}$ are $s=\sqrt{r^{2}+z^{2}}$ and $s^{\prime}=\sqrt{|\vec{b}-\vec{r}|^{2}+z^{\prime 2}}$. In the absence of nuclear modifications, $S^{i}\left(A, x, Q^{2}, \vec{r}, z\right) \equiv 1$. The nuclear density distribution is described by a Woods-Saxon parameterization,

$$
\rho_{A}(s)=\rho_{0} \frac{1+\omega\left(s / R_{A}\right)^{2}}{1+\exp \left[\left(s-R_{A}\right) / d\right]} .
$$

Electron scattering data [11] are used to fix the parameters $R_{A}, d, \omega$ and $\rho_{0}$.

Experiments [12] have shown that the proton and neutron structure functions are modified in the nucleus. For momentum fractions $x<0.1$ and $0.3<x<0.7$, a depletion is observed in a heavy nucleus relative to a light nucleus such as the deuteron. The low $x$, shadowing, region and the larger $x$, EMC, region is bridged by an enhancement at $0.1<x<0.3$ called antishadowing. Here we refer to the modification over the entire $x$ range as 'shadowing' unless otherwise noted. Many theoretical explanations have been proposed, typically for only part of the $x$ range such as the very low $x$ or EMC regions. However, as none of the models can describe the effect over all $x$ and $Q^{2}$, we rely on parameterizations of the nuclear modifications based on fits to data, as described later.

Most typical structure function measurements are insensitive to any spatial dependence and thus average over the entire nuclear volume. One experiment using a bubble chamber found that the structure function does vary spatially but could not determine the dependence on impact parameter [13]. In a nuclear collision, the impact parameter can be determined from the transverse energy production. The influence of the spatial dependence of shadowing on transverse energy production has already been considered [2,14]. The effects of spatially inhomogeneous shadowing on heavy quark [9, 15], quarkonium, and Drell-Yan [2,16] production in heavy ion collisions has also been discussed previously.

We now describe the NLO cross section in Eq. (1) in more detail. The functions $H_{i j}^{V}$ are rather simple [17]:

$$
H_{i j}^{Z^{0}}=\frac{8 \pi}{3} \frac{G_{F}}{\sqrt{2}}\left[\left(g_{V}^{i}\right)^{2}+\left(g_{A}^{i}\right)^{2}\right] \frac{m_{Z}^{2}}{s}
$$




$$
H_{i j}^{W^{ \pm}}=\frac{2 \pi}{3} \frac{G_{F}}{\sqrt{2}} \frac{m_{W}^{2}}{s}
$$

where $G_{F}=1.16639 \times 10^{-5} \mathrm{GeV}^{2}, m_{Z}=91.187 \mathrm{GeV}$, and $m_{W}=80.41 \mathrm{GeV}$. For $Z^{0}$ production by a given flavor $i$ with charge $e_{i}$, the sum of the squared vector and axial vector couplings is $\left(g_{V}^{i}\right)^{2}+\left(g_{A}^{i}\right)^{2}=(1 / 8)\left(1-4\left|e_{i}\right| x_{W}+8 e_{i}^{2} x_{W}^{2}\right)$ where $x_{W}=\sin ^{2} \theta_{W}=1-m_{W}^{2} / m_{Z}^{2}$.

The functions $\Delta_{i j}(x)$ are universal for all vector bosons, including virtual photons produced in the Drell-Yan process [17]. We work in the $\overline{\mathrm{MS}}$ scheme. The NLO correction to the $q \bar{q}$ channel includes the contributions from soft and virtual gluons as well as hard gluons from the process $q \bar{q} \rightarrow V g$. We have, up to NLO [17],

$$
\begin{aligned}
\Delta_{q \bar{q}}(x)= & \delta(1-x)+\frac{\alpha_{s}\left(Q^{2}\right)}{3 \pi}\left\{-4(1+x) \ln \left(\frac{Q^{2}}{m_{V}^{2}}\right)-8(1+x) \ln (1-x)-4 \frac{1+x^{2}}{1-x} \ln x\right. \\
& \left.+\delta(1-x)\left[6 \ln \left(\frac{Q^{2}}{m_{V}^{2}}\right)+8 \zeta(2)-16\right]+8\left[\frac{1}{1-x}\right]_{+} \ln \left(\frac{Q^{2}}{m_{V}^{2}}\right)+16\left[\frac{\ln (1-x)}{1-x}\right]_{+}\right\} .
\end{aligned}
$$

The first delta function is the LO contribution while the NLO contribution is proportional to $\alpha_{s}\left(Q^{2}\right)$. At NLO $\alpha_{s}\left(Q^{2}\right)$ is calculated to two loops with $n_{f}=5$ active flavors. The last three terms are the soft and virtual gluon contributions. The general integral of the 'plus' functions in the last two terms is 18

$$
\int_{a}^{1} d x f(x)\left[\frac{\ln ^{i}(1-x)}{1-x}\right]_{+}=\int_{a}^{1} d x \frac{f(x)-f(1)}{1-x} \ln ^{i}(1-x)+\frac{f(1)}{i+1} \ln ^{i+1}(1-a) .
$$

The quark-gluon contribution only appears at $\mathcal{O}\left(\alpha_{s}\right)$ through the real correction $q g \rightarrow q V$. At this order [17],

$$
\Delta_{q g}(x)=\frac{\alpha_{s}\left(Q^{2}\right)}{8 \pi}\left\{2\left(1+2 x^{2}-2 x\right) \ln \left(\frac{(1-x)^{2} Q^{2}}{x m_{V}^{2}}\right)+1-7 x^{2}+6 x\right\} .
$$

For gauge boson production, we take $Q^{2}=m_{V}^{2}$ and all terms proportional to $\ln \left(Q^{2} / m_{V}^{2}\right)$ drop out. Using the delta functions in Eq. (1) we find $x_{1,2}=\left(m_{V} / \sqrt{x s}\right) \exp ( \pm y)$. As at LO, when $\Delta_{q \bar{q}}(x)$ is proportional to $\delta(1-x)$ in Eq. (6),$x_{1,2}^{\prime}=\left(m_{V} / \sqrt{s}\right) \exp ( \pm y)$. The rather lengthy convolutions of the shadowing functions and parton distribution functions including isospin via the proton and neutron numbers are given in the appendix.

We now define the coupling matrices in Eq. (1). The superscripts represent the initial (i) and final (f) state quarks or antiquarks while the arguments indicate the orientation of the quark line to which the boson is coupled [17]. The coupling matrices are fairly simple for $Z^{0}$ production: $C^{\mathrm{ii}}\left(q_{i}, \bar{q}_{j}\right)=\delta_{i j}$ and $C^{\mathrm{if}}\left(q_{i}, q_{k}\right)=\delta_{i k}$. With $W^{+}$and $W^{-}$production, the couplings are elements of the CKM matrix. They are nonzero for $C^{\mathrm{ii}}\left(q_{k}, \bar{q}_{l}\right)$ if $e_{k}+e_{l}= \pm 1$ and for $C^{\text {if }}\left(q_{k}, q_{l}\right)$ if $e_{k}= \pm 1+e_{l}$. In both cases, they take the values $\left|V_{q_{k} q_{l}}\right|^{2}$. Following Hamberg et al. [17], we take $V_{u d}=\cos \theta_{C} \approx V_{c s}$ and $V_{u s}=\sin \theta_{C} \approx-V_{c d}$ with $\sin \theta_{C} \approx 0.22$.

We use the MRST HO (central gluon) [19] nucleon parton distributions in the $\overline{\mathrm{MS}}$ scheme, shown evaluated at $Q^{2}=m_{Z}^{2}$ in Fig. 1. The valence distributions are somewhat larger than the corresponding sea quark distributions at $x \geq 0.1$ and extend to higher $x$ values. The sea quarks dominate the valence quarks at $x \sim 10^{-4}$ by a factor of $\sim 100$. Note also that $f \frac{p}{d}$ is larger than $f_{\bar{u}}$ when $x>0.01$. The gluon distribution is shown at $1 / 10$ of its magnitude. At low $x$, corresponding to large rapidity, the gluon density is high. 
The shadowing effect is studied with three parameterizations of the average, homogeneous, shadowing, $S_{k}^{i}\left(A, x, Q^{2}\right)\{k=1-3\}$, measured in nuclear deep-inelastic scattering. All the shadowing parameterizations are obtained and evolved at leading order. Since the parameterizations are fit to ratios of heavy to light nuclei, the dependence of the parameterizations on both the initial parton densities and the order of the calculation should be weak. The first, $S_{1}(A, x)$, assumes that the quark, gluon and antiquark modifications are equivalent and includes no $Q^{2}$ evolution [20]. The second, $S_{2}^{i}\left(A, x, Q^{2}\right)$, has separate modifications for the valence quarks, sea quarks and gluons and includes $Q^{2}$ evolution from $4<Q^{2}<100 \mathrm{GeV}^{2}$ [21]. The third parameterization, $S_{3}^{i}\left(A, x, Q^{2}\right)$, is based on the GRV LO [22] parton densities. The ratios are evolved over $2.25<Q^{2}<10^{4} \mathrm{GeV}^{2}$ [23,24] assuming that $S_{3}^{u_{V}}=S_{3}^{d_{V}}$ and $S_{3}^{\bar{u}}=S_{3}^{\bar{d}}$ while the more massive sea quarks are evolved separately. Both the $S_{2}$ and $S_{3}$ ratios are evolved to higher $Q^{2}$ using the DGLAP equations [21,23, 24]. It was shown in Ref. [21] that including recombination terms in the evolution, as in Ref. [25], did not have a large effect on the shadowing ratios, particularly at the $x$ values probed here. The initial gluon ratio in $S_{3}$ shows significant antishadowing for $0.1<x<0.3$ while the sea quark ratios are shadowed. In contrast, $S_{2}$ has less gluon antishadowing and essentially no sea quark effect in the same $x$ region. Unfortunately, the $Q^{2}$ evolution of $S_{2}$ stops below the vector boson mass, rendering it less valuable. We show results with all three parameterizations because no nuclear DIS data is available at high $Q^{2}$. Since the $S_{3}$ parameterization includes the most recent nuclear DIS data and is evolved to scales compatible with the vector boson masses, the $S_{3}$ results should perhaps be favored.

The shadowing ratios in a lead nucleus compared to a proton are shown in Fig. 2. The effects of shadowing on the valence quarks is strongest with $S_{1}$ since all quarks are treated equally. The $S_{2}$ and $S_{3}$ valence ratios are rather similar in magnitude although the antishadowing range is broadest for $S_{3}, 0.01<x<0.3$ and the $S_{3}$ ratio is lower than the $S_{2}$ ratio at low $x$. The $S_{1}$ and $S_{2}$ sea quark ratios are very similar when $x<0.1$. Then the $S_{2}$ ratio is essentially unity until $x>0.3$. The $S_{3}$ ratios are all larger than the $S_{1}$ and $S_{2}$ ratios, even at small $x$, due to evolution. It is most interesting to note the difference between the light and strange sea ratios in the $S_{3}$ parameterization. The ratios $S_{3}^{\bar{u}}$ and $S_{3}^{\bar{d}}$ show no antishadowing effect but instead decrease when $x>0.1$ while $S_{3}^{\bar{s}}$ and $S_{3}^{\bar{c}}$ are typically larger over all $x$ and are antishadowed when $0.01<x<0.2$. The antishadowing in the charm distribution is larger even than for the gluons. Since all $S_{3}$ sea ratios are equivalent at $Q^{2}=2.25 \mathrm{GeV}^{2}$, the difference is solely the effect of evolution. Note also that the gluon shadowing ratios are typically larger than the large $Q^{2}$ sea quark ratios over all $x$. At $Q^{2}=m_{Z}^{2}$ the strong antishadowing in $S_{3}^{g}$ has essentially disappeared and is no larger than that of $S_{2}^{g}$ although the antishadowing region of $S_{3}^{g}$ is broader, from $0.005<x<0.2$.

Nuclear shadowing should depend on spatial position of the partons in the nucleus as well as on their momentum. Most models predict some form of spatial dependence, according to the origin of the shadowing effect. Typically, the spatial dependence can be expected to take two forms, either proportional to the local nuclear density, Eq. (3), or the path length of the parton through the nucleus. Both will be discussed below.

When the parton density is high, partons in one nucleon can interact with those in neighboring nucleons, recombining to lower the parton density [26]. In this case shadowing is proportional to the local nuclear density [9, [15]. Then 


$$
S_{k \mathrm{WS}}^{i}=S_{k}^{i}\left(A, x, Q^{2}, \vec{r}, z\right)=1+N_{\mathrm{WS}}\left[S_{k}^{i}\left(A, x, Q^{2}\right)-1\right] \frac{\rho_{A}(s)}{\rho_{0}},
$$

where $N_{\mathrm{WS}}$ is a normalization constant chosen so that $(1 / A) \int d^{3} s \rho_{A}(s) S_{k \mathrm{WS}}^{i}=S_{k}^{i}$. At large radii, $s \gg R_{A}$, the medium modifications weaken and the nucleons behave as though they were free. At the center of the nucleus, the modifications are larger than the average value determined from nuclear DIS.

It has also been suggested that shadowing stems from multiple interactions of the incident parton [27]. In this picture, parton-parton interactions are longitudinally distributed over the coherence length, $l_{c}=1 / 2 m_{N} x$, where $m_{N}$ is the nucleon mass [28]. When $x<0.016$, $l_{c}>R_{A}$ for all nuclei and the interaction of the initial parton is delocalized over the entire nuclear path, thus interacting coherently with all target partons along the distance $l_{c}$. For small $x$, shadowing depends on the longitudinally-integrated nuclear density at transverse distance $\vec{r}$ and the spatial dependence can then be parameterized as

$$
S_{k \rho}^{i}\left(A, x, Q^{2}, \vec{r}, z\right)=1+N_{\rho}\left(S_{k}^{i}\left(A, x, Q^{2}\right)-1\right) \frac{\int d z \rho_{A}(\vec{r}, z)}{\int d z \rho_{A}(\overrightarrow{0}, z)}
$$

where the normalization is again defined by $(1 / A) \int d^{2} r d z \rho_{A}(s) S_{k \rho}^{i}=S_{k}^{i}$ with $N_{\rho}>N_{\text {Ws }}$. However, at large $x, l_{c} \ll R_{A}$ and shadowing is again proportional to the local density so that Eq. (9) corresponds to the large $x$ limit of the multiple scattering formulation.

There are some problems with implementing the multiple scattering picture in nuclear collisions. While traversing the formation length, both the initial- and final-state partons may undergo multiple interactions, reducing the effective $l_{c}$, similar to the LandauPomeranchuk-Migdal effect [29]. In addition, the idealized picture of a single initial parton incident on a static nucleus is inappropriate in heavy ion collisions since many interactions occur simultaneously, increasing the density in the path of the initial parton. A cascade approach cannot resolve the difficulty because non-local depictions of these collisions are Lorentz frame dependent [30]. Finally, since the parton densities are distributed over an $x$-dependent distance, baryon number is not locally conserved even if the valence quarks are considered to be fixed spatially. Given the difficulties with the multiple interaction picture as well as those of matching the two spatial dependencies according to $l_{c}$ at each $x$, we only present specific results for the local density model, Eq. (9).

Other mechanisms of shadowing effects in the EMC region such as nuclear binding [31] and rescaling [32.33] have also been suggested but can explain only part of the observed effect [34. We note that these models would also predict some spatial dependence.

We first show that our shadowing results do not depend strongly on the order of the calculation. The ratio of the NLO to LO cross sections, both calculated with the MRST HO distributions, is often referred to as the $K$ factor. The $K$ factor is given as a function of rapidity with no shadowing for $\mathrm{Pb}+\mathrm{Pb}$ collisions at $5.5 \mathrm{TeV}$ in Fig. 通 for all three vector bosons. The $K$ factor is $\approx 1.13$ up to $y=3$. It grows larger as the edge of phase space is approached since large, positive, $y$ corresponds to low $x_{2}$ where the gluon density is high and the $q g$ channel becomes more important. The $q g$ channel contributes $\approx 15 \%$ of the total vector boson cross section, with or without shadowing. The $K$ factor increases faster with $y$ for the $Z^{0}$ because the higher mass means that the phase space for $Z^{0}$ production is exhausted at lower rapidities than $W^{ \pm}$production, leading to larger $q g$ contributions at 
high $y_{Z}$. The $K$ factors are quite similar when shadowing is included and differ from those without shadowing by $\approx 1 \%$ at $y=0$. At high rapidities, a larger effect might be expected from the gluons but, as seen in Fig. 2(c), the effects of shadowing on the gluon distributions are not as strong as those of the sea quarks at large $Q^{2}$. Thus the difference in the $K$ factors between the calculations with and without shadowing is only marginally larger at high rapidity, up to $\approx 4 \%$ at $y=4$.

In Fig. 4, we show the ratio of $Z^{0}$ production in $\mathrm{Pb}+\mathrm{Pb}$ collisions with and without shadowing at both LO and NLO. The results are independent of the order of the calculation, even at large rapidities. This is not surprising since we have shown that the differences between shadowing ratios at LO and NLO are trivial for virtual photon production via the Drell-Yan process [2], even for pairs with $m<m_{\Upsilon}$. At the higher scale of $Z^{0}$ and $W^{ \pm}$ production, the approximation should be even better because the $K$ factor is smaller.

We now calculate the NLO $Z^{0}, W^{+}$and $W^{-}$cross sections in nuclear collisions. Table — gives the total cross sections in the CMS and ALICE central acceptances, $|y|<2.4$ and $|y|<$ 1 respectively, at the LHC. The cross sections are larger than the virtual photon mediated Drell-Yan cross sections at lower masses [2]. The results, given for $\mathrm{Pb}+\mathrm{Pb}$ collisions, are integrated over impact parameter and presented in units of nb/nucleon pair. We note that with the normalization of $S_{\mathrm{WS}}$, the impact-parameter integrated cross section is unchanged when the spatial dependence is included. The next-to-next-to-leading order, NNLO, $W^{ \pm}$ and $Z^{0}$ total cross sections have also been calculated [17]. The $K$ factors obtained from the ratio of the $\mathcal{O}\left(\alpha \alpha_{s}^{2}\right)$ to $\mathcal{O}(\alpha)$ cross sections differs by $\approx 1 \%$ from the $\mathcal{O}\left(\alpha \alpha_{s}\right)$ to $\mathcal{O}(\alpha) K$ factor shown in Fig. 3. Thus changes in the total cross sections between NLO and NNLO are on the few percent level even though the vector bosons can be produced in the $g g$ channel as well at NNLO, because $\alpha_{s}\left(m_{V}^{2}\right) \approx 0.116$. The effects on the shadowing ratios should be even smaller, see Fig. 1.

We have checked how our results depend on the chosen set of parton densities. Using

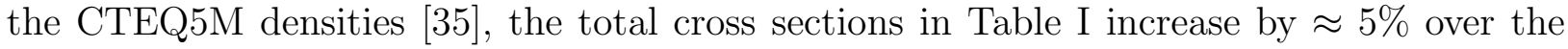
MRST HO results. However, the $K$ factors and shadowing ratios in Figs. 3 and 1 change by less than $1 \%$. Thus our shadowing results are essentially independent of parton density.

In Table II we show the expected rate in nucleus-nucleus collisions at $b=0, N\left(S=S_{k}\right)=$ $\sigma_{N N} T_{\mathrm{PbPb}}(0) L_{\mathrm{PbPb}}^{\text {int }} \sigma\left(S=S_{k}\right)$ with $L_{\mathrm{PbPb}}^{\text {int }}=1 / \mathrm{nb}$ in a one month $\left(10^{6} \mathrm{~s}\right)$ LHC run, $\sigma_{N N}=60$ $\mathrm{mb}$ at LHC energies, and $T_{\mathrm{PbPb}}(0)=30.4 / \mathrm{mb}$. The absolute numbers in the experimental acceptances are large but do not reflect the measurable decay channels. Including the 3.37\% lepton pair branching for $Z^{0}$ decays reduces the number produced with no shadowing, $S=1$, to 990 in CMS and 425 in ALICE. The 10\% lepton branching ratio for $W^{+}$and $W^{-}$leaves nearly 4600 observable decays in CMS and 1980 in ALICE.

Figures 5 and 6 compare the ratios of $Z^{0}$ and $W^{+}$production in $\mathrm{Pb}+\mathrm{Pb}$ collisions with the three shadowing parameterizations to $\mathrm{Pb}+\mathrm{Pb}$ collisions with no shadowing as a function of rapidity. The isospin effects wash out the differences between the $W^{+}$and $W^{-}$distributions in the ratios so that the results are essentially identical for the two charged vector bosons. Therefore the ratios are shown only for the $W^{+}$. The results are given for several impact parameter bins, the most central bin, $b<0.2 R_{A}$, an intermediate impact parameter bin around $b \sim R_{A}$, and a peripheral bin around $b \sim 2 R_{A}$. It is clear that by neglecting the impact parameter dependence of shadowing, one may overestimate the effect in peripheral collisions, an important point if using the $Z^{0}$ as a baseline in different transverse energy 
bins. The integration over all impact parameters is equivalent to the average shadowing, as expected from the normalization of Eq. (9). Although the results are shown using the local density approximation, the parameterization of the spatial dependence for a long coherence length, Eq. (10), differs only marginally. In central collisions, the difference between the two parameterizations is less than $1 \%$ while in the most peripheral bin, it is $3-6 \%$. The largest differences occur in regions with the strongest shadowing modifications. Thus the calculations are rather insensitive to the exact spatial parameterization, suggesting that heavy ion collisions cannot distinguish between different dependencies, only between homogeneous and inhomogeneous shadowing.

The ratios are rather similar for all vector bosons. The $S_{1}$ and $S_{2}$ ratios are approximately equal as a function of rapidity, presumably because the $Q^{2}$ evolution of the $S_{2}$ parameterization ends at $Q^{2}=100 \mathrm{GeV}^{2}$. The calculations cover the entire rapidity range of vector boson production. At $y_{Z} \sim 0, x_{1}=x_{2}=0.017$, in the low $x$ region. As rapidity increases, $x_{1}$ increases, going through the antishadowing region and the EMC region with $x_{1} \sim 0.33$ at $y_{Z}=3$. The kink in the $S_{1}$ ratio at $y \sim 2.2$ is an artifact resulting from the rather sharp transition between the shadowing and EMC regions at $x \sim 0.15$, see Fig. 2. Note that the larger rapidity coverage of CMS makes the EMC region accessible in this measurement. When $y_{Z} \rightarrow 4, x_{1} \rightarrow 1$, entering the "Fermi motion" region and causing the upturn of the ratios at large $y_{Z}$. Note also that at large $x_{1}$, the valence quarks dominate. While increasing $y_{Z}\left(x_{1}\right)$ traces out the large $x$ portion of the shadowing curve, the low $x$ part of the shadowing regime is accessible from $x_{2}$ with growing $y_{Z}$. At $y_{Z}=3, x_{2} \sim 8 \times 10^{-4}$, in a range where shadowing saturates in $S_{1}$ and $S_{2}$. There is no saturation built into the $S_{3}$ parameterization, causing a steeper decrease in the ratios for large $y_{Z}$ with this parameterization than with $S_{1}$ and $S_{2}$. In addition, the $S_{3}$ sea quark shadowing is never as strong at low $x$ as for $S_{1}$ and $S_{2}$ so that these two parameterizations are both more strongly shadowed overall. The $Z^{0}$ ratios are all slightly higher than those for $W^{ \pm}$because the larger mass of the $Z^{0}$ results in $x_{Z} \sim 1.1 x_{W}$.

We also point out that the large vector boson masses do not allow us to restrict ourselves only to an $x$ region where the coherence length is always larger than $R_{A}$ so that the multiple interaction approach could be used without having to match the spatial dependence across $x$ boundaries. While the target parton is at relatively low $x$, the projectile parton, also affected by shadowing, is at relatively high $x$ where $l_{c}$ is small.

The shadowing ratios are fairly simply traced out for vector boson production, especially at leading order since the fixed boson mass defines $x$ at any $y$ whereas Drell-Yan shadowing effects are smeared over the mass interval. However, the ratios shown in Figs. 5 and 6 will not be accessible experimentally due to the nuclear isospin. The comparison must be made to $p p$ interactions, preferably at the same energy to retain the same $x$ values. This ideal situation may not be realized for some time at the LHC. Therefore in Figs. 강 we show the $\mathrm{Pb}+\mathrm{Pb}$ rapidity distributions with and without homogeneous shadowing as well as the distributions from $p p$ collisions at $14 \mathrm{TeV}$ for all three vector bosons. The $\mathrm{Pb}+\mathrm{Pb}$ cross sections are given per nucleon pair for a more direct comparison. The higher energy extends the available vector boson rapidity space by one unit. The $Z^{0}$ distributions in Fig. 7 have a plateau over two units of rapidity. The $p p W^{+}$distribution in Fig. 8 rises over the first several units of rapidity, followed by a decrease as the edge of phase space is approached. This is due to the increasing importance of valence quarks at large $y$ (large $x_{1}$ ). The effect appears for $W^{+}$ 
production in $p p$ collisions because the $u$ valence quarks carry more momentum than the $d$ valence quarks, see Fig. 1 (a). On the other hand, the $W^{-} p p$ distribution always decreases with rapidity. In $\mathrm{Pb}+\mathrm{Pb}$ collisions, there is a slight increase in $W^{-}$production with rapidity instead of a decrease while the $W^{+}$distributions are either flat or decreasing. This increase in $W^{-}$production shown in Fig. 9 is due to the neutron excess in $\mathrm{Pb}+\mathrm{Pb}$ where, in $n n$ collisions, $W^{-}$production proceeds dominantly through $f_{u}^{n} f_{d}^{n}\left(\approx f_{\frac{p}{d}}^{p} f_{u}^{p}\right)$. Likewise, the rise in $W^{+}$production in $\mathrm{Pb}+\mathrm{Pb}$ relative to $p p$ collisions disappears because of the neutron content of the nucleus.

Finally, the ratios of the $\mathrm{Pb}+\mathrm{Pb} /$ nucleon pair to $p p$ cross sections are shown in Figs. I012 for homogeneous shadowing. Due to the higher $p p$ cross sections, the ratios are lower than those to $S=1$ at $5.5 \mathrm{TeV}$ shown in Figs. 5 and 6 . Since both the $\mathrm{Pb}+\mathrm{Pb}$ and $p p Z^{0}$ rapidity distributions are rather flat, the ratios in Fig. 10 are also flat to $y \sim 1.5$. The rise in the $W^{+} p p$ distribution shown in Fig. 8 causes the $\mathrm{Pb}+\mathrm{Pb}$ to $p p$ ratios to decrease with rapidity over all $y$ in Fig. 11. However, the increase in $W^{-}$production due to neutrons in $\mathrm{Pb}+\mathrm{Pb}$ collisions, barely visible in Fig. 9, is apparent in the $W^{-} \mathrm{Pb}+\mathrm{Pb}$ to $p p$ ratio in Fig. 12. It should still be possible to distinguish between the shadowing parameterizations and study quark shadowing at $Q^{2}=m_{V}^{2}$, particularly since the $14 \mathrm{TeV} p p$ data will be available with higher statistics. Note that comparing the $p p$ results to the $\mathrm{Pb}+\mathrm{Pb}$ calculations with inhomogeneous shadowing would result in slightly lower ratios in central collisions and higher ratios in peripheral collisions, as expected from Figs. 5 and 6 .

Once the basic nuclear shadowing effects on vector boson production have been understood, they can perhaps be used to study other medium effects in heavy ion collisions by comparing the leptonic and hadronic decay channels. The hadronic decays of the vector bosons, $\sim 70 \%$ of all decays of each boson, may be more difficult to interpret. While the width of the $Z^{0}$ decay to $l^{+} l^{-}$is not expected to be modified in the quark-gluon plasma due to the weak coupling [36], the $Z^{0}$ has a $2.49 \mathrm{GeV}$ total width and will decay in any quarkgluon plasma to two jets through $Z^{0} \rightarrow q \bar{q} \rightarrow$ jet + jet in $\sim 0.1 \mathrm{fm}$. Therefore, the decay jets could be modified in the medium which may still be progressing toward thermalization and will be subject to rescattering and jet quenching. Thus a comparison of a reconstructed $Z^{0}$ in the dilepton channel where no nuclear effects are expected and medium-modified jets should result in a broader width in the $q \bar{q}$ channel than the $l^{+} l^{-}$channel [37. In addition, the $Z^{0}$ could be used to tag jets through the $q \bar{q} \rightarrow Z^{0} g$ and $g q \rightarrow Z^{0} q$ channels to study jet properties in the quark-gluon plasma 8 .

Acknowledgements I thank K.J. Eskola for providing the shadowing parameterizations. I thank D. Kharzeev, K. Redlich and U.A. Wiedemann for discussions.

\section{Appendix}

In $A B$ collisions, the cross section per nucleon must include the nuclear isospin since, in general, $\sigma_{p p}^{V} \neq \sigma_{p n}^{V} \neq \sigma_{n p}^{V} \neq \sigma_{n n}^{V}$. We give the convolution of the nuclear parton densities in Eq. (1), including only the couplings. We take $f_{d_{V}}^{n}=f_{u_{V}}^{p}, f_{u_{V}}^{n}=f_{d_{V}}^{p}, f_{\bar{d}}^{n}=f_{\frac{u}{u}}^{p}$, and $f_{\bar{u}}^{n}=f_{\bar{d}}^{p}$. All other distributions are assumed to be identical for protons and neutrons. The proton and neutron numbers in nucleus $A$ are $Z_{A}$ and $N_{A}$. To be concise, we define

$$
\begin{aligned}
& S^{u}(A, x) f_{u}^{p}\left(x, Q^{2}\right)=S^{u_{V}}(A, x) f_{u_{V}}^{p}+S^{\bar{u}}(A, x) f_{\bar{u}}^{p} \\
& S^{d}(A, x) f_{d}^{p}\left(x, Q^{2}\right)=S^{d_{V}}(A, x) f_{d_{V}}^{p}+S^{\bar{d}}(A, x) f_{\bar{d}}^{p}
\end{aligned}
$$


where we have abbreviated the shadowing functions as $S^{i}(A, x)$.

We begin with the $q \bar{q}$ channel. For $Z^{0}$ production, we have

$$
\begin{aligned}
& \sum_{i, j \in Q \bar{Q}} S^{i}\left(A, x_{1}\right) S^{j}\left(B, x_{2}\right) f_{q_{i}}^{N}\left(x_{1}, Q^{2}\right) f_{\bar{q}_{j}}^{N}\left(x_{2}, Q^{2}\right) C^{\mathrm{ii}}\left(q_{i}, \bar{q}_{j}\right)\left[\left(g_{V}^{i}\right)^{2}+\left(g_{A}^{i}\right)^{2}\right] \\
& =\frac{1}{8}\left[1-\frac{8}{3} x_{W}+\frac{32}{9} x_{W}^{2}\right]\left(S^{u}\left(A, x_{1}\right) S^{\bar{u}}\left(B, x_{2}\right)\left\{Z_{A} f_{u}^{p}\left(x_{1}, Q^{2}\right)+N_{A} f_{u}^{n}\left(x_{1}, Q^{2}\right)\right\}\right. \\
& \left.\times\left\{Z_{B} f_{\bar{u}}^{p}\left(x_{2}, Q^{2}\right)+N_{B} f_{\bar{u}}^{n}\left(x_{2}, Q^{2}\right)\right\}+2 A B S^{c}\left(A, x_{1}\right) S^{\bar{c}}\left(B, x_{2}\right) f_{c}^{p}\left(x_{1}, Q^{2}\right) f_{\bar{c}}^{p}\left(x_{2}, Q^{2}\right)\right) \\
& +\frac{1}{8}\left[1-\frac{4}{3} x_{W}+\frac{8}{9} x_{W}^{2}\right]\left(S^{d}\left(A, x_{1}\right) S^{\bar{d}}\left(B, x_{2}\right)\left\{Z_{A} f_{d}^{p}\left(x_{1}, Q^{2}\right)+N_{A} f_{d}^{n}\left(x_{1}, Q^{2}\right)\right\}\right. \\
& \left.\times\left\{Z_{B} f_{\bar{d}}^{p}\left(x_{2}, Q^{2}\right)+N_{B} f_{\bar{d}}^{n}\left(x_{2}, Q^{2}\right)\right\}+2 A B S^{s}\left(A, x_{1}\right) S^{\bar{s}}\left(B, x_{2}\right) f_{s}^{p}\left(x_{1}, Q^{2}\right) f_{\bar{s}}^{p}\left(x_{2}, Q^{2}\right)\right) \\
& +\left[x_{1} \leftrightarrow x_{2}, A \leftrightarrow B\right] .
\end{aligned}
$$

Note that for $Z^{0}$ production, we have also included the square of the vector and axial vector couplings since these depend on the quark charges. The $W^{+} q \bar{q}$ convolution is

$$
\begin{aligned}
& \sum_{i, j \in Q, \bar{Q}} S^{i}\left(A, x_{1}\right) S^{j}\left(B, x_{2}\right) f_{q_{i}}^{N}\left(x_{1}, Q^{2}\right) f_{\bar{q}_{j}}^{N}\left(x_{2}, Q^{2}\right) C^{\mathrm{ii}}\left(q_{i}, \bar{q}_{j}\right) \\
& =\cos ^{2} \theta_{C}\left(S^{u}\left(A, x_{1}\right) S^{\bar{d}}\left(B, x_{2}\right)\left\{Z_{A} f_{u}^{p}\left(x_{1}, Q^{2}\right)+N_{A} f_{u}^{n}\left(x_{1}, Q^{2}\right)\right\}\right. \\
& \left.\times\left\{Z_{B} f_{\frac{p}{d}}\left(x_{2}, Q^{2}\right)+N_{B} f_{\bar{d}}^{n}\left(x_{2}, Q^{2}\right)\right\}+A B S^{\bar{s}}\left(A, x_{1}\right) S^{c}\left(B, x_{2}\right) f_{\bar{s}}^{p}\left(x_{1}, Q^{2}\right) f_{c}^{p}\left(x_{2}, Q^{2}\right)\right) \\
& +\sin ^{2} \theta_{C}\left(S^{u}\left(A, x_{1}\right) S^{\bar{s}}\left(B, x_{2}\right)\left\{Z_{A} f_{u}^{p}\left(x_{1}, Q^{2}\right)+N_{A} f_{u}^{n}\left(x_{1}, Q^{2}\right)\right\} B f_{\bar{s}}^{p}\left(x_{2}, Q^{2}\right)\right. \\
& \left.\left.+S^{\bar{d}}\left(A, x_{1}\right) S^{c}\left(B, x_{2}\right)\left\{Z_{A} f_{\bar{d}}^{p}\left(x_{1}, Q^{2}\right)+N_{A} f_{\bar{d}}^{n}\left(x_{1}, Q^{2}\right)\right\} B f_{c}^{p}\left(x_{2}, Q^{2}\right)\right)\right\} \\
& +\left[x_{1} \leftrightarrow x_{2}, A \leftrightarrow B\right] .
\end{aligned}
$$

Finally, the $W^{-} q \bar{q}$ convolution is

$$
\begin{aligned}
& \sum_{i, j \in Q \bar{Q}} S^{i}\left(A, x_{1}\right) S^{j}\left(B, x_{2}\right) f_{q_{i}}^{N}\left(x_{1}, Q^{2}\right) f_{\bar{q}_{j}}^{N}\left(x_{2}, Q^{2}\right) C^{\mathrm{ii}}\left(q_{i}, \bar{q}_{j}\right) \\
& =\cos ^{2} \theta_{C}\left(S^{\bar{u}}\left(A, x_{1}\right) S^{d}\left(B, x_{2}\right)\left\{Z_{A} f_{\bar{u}}^{p}\left(x_{1}, Q^{2}\right)+N_{A} f_{\bar{u}}^{n}\left(x_{1}, Q^{2}\right)\right\}\right. \\
& \left.\times\left\{Z_{B} f_{d}^{p}\left(x_{2}, Q^{2}\right)+N_{B} f_{d}^{n}\left(x_{2}, Q^{2}\right)\right\}+A B S^{s}\left(A, x_{1}\right) S^{\bar{c}}\left(B, x_{2}\right) f_{s}^{p}\left(x_{1}, Q^{2}\right) f_{\bar{c}}^{p}\left(x_{2}, Q^{2}\right)\right) \\
& +\sin ^{2} \theta_{C}\left(S^{\bar{u}}\left(A, x_{1}\right) S^{s}\left(B, x_{2}\right)\left\{Z_{A} f_{\bar{u}}^{p}\left(x_{1}, Q^{2}\right)+N_{A} f_{\bar{u}}^{n}\left(x_{1}, Q^{2}\right)\right\} B f_{s}^{p}\left(x_{2}, Q^{2}\right)\right. \\
& \left.\left.+S^{d}\left(A, x_{1}\right) S^{\bar{c}}\left(B, x_{2}\right)\left\{Z_{A} f_{d}^{p}\left(x_{1}, Q^{2}\right)+N_{A} f_{d}^{n}\left(x_{1}, Q^{2}\right)\right\} B f_{\bar{c}}^{p}\left(x_{2}, Q^{2}\right)\right)\right\} \\
& +\left[x_{1} \leftrightarrow x_{2}, A \leftrightarrow B\right] .
\end{aligned}
$$

We now turn to the $q g$ channel. The convolution for $Z^{0}$ production is

$$
\begin{aligned}
& \sum_{i, k \in Q \bar{Q}}\left(S^{i}\left(A, x_{1}\right) S^{g}\left(B, x_{2}\right) f_{q_{i}}^{N}\left(x_{1}, Q^{2}\right) f_{g}^{N}\left(x_{2}, Q^{2}\right)\right. \\
& \left.+\left[x_{1} \leftrightarrow x_{2}, A \leftrightarrow B\right]\right) C^{\mathrm{if}}\left(q_{i}, q_{k}\right)\left[\left(g_{V}^{i}\right)^{2}+\left(g_{A}^{i}\right)^{2}\right] \\
& =B S^{g}\left(B, x_{2}\right) f_{g}^{p}\left(x_{2}, Q^{2}\right)\left\{\frac { 1 } { 8 } [ 1 - \frac { 8 } { 3 } x _ { W } + \frac { 3 2 } { 9 } x _ { W } ^ { 2 } ] \left(S^{u}\left(A, x_{1}\right)\left\{Z_{A} f_{u}^{p}\left(x_{1}, Q^{2}\right)+N_{A} f_{u}^{n}\left(x_{1}, Q^{2}\right)\right\}\right.\right.
\end{aligned}
$$




$$
\begin{aligned}
& \left.+S^{\bar{u}}\left(A, x_{1}\right)\left\{Z_{B} f_{\bar{u}}^{p}\left(x_{2}, Q^{2}\right)+N_{B} f_{\bar{u}}^{n}\left(x_{2}, Q^{2}\right)\right\}+2 A S^{c}\left(A, x_{1}\right) f_{c}^{p}\left(x_{1}, Q^{2}\right)\right) \\
& +\frac{1}{8}\left[1-\frac{4}{3} x_{W}+\frac{8}{9} x_{W}^{2}\right]\left(S^{d}\left(A, x_{1}\right)\left\{Z_{A} f_{d}^{p}\left(x_{1}, Q^{2}\right)+N_{A} f_{d}^{n}\left(x_{1}, Q^{2}\right)\right\}\right. \\
& \left.\left.+S^{\bar{d}}\left(A, x_{1}\right)\left\{Z_{B} f_{\bar{d}}^{p}\left(x_{2}, Q^{2}\right)+N_{B} f_{\bar{d}}^{n}\left(x_{2}, Q^{2}\right)\right\}+2 A S^{s}\left(A, x_{1}\right) f_{s}^{p}\left(x_{1}, Q^{2}\right)\right)\right\} \\
& +\left[x_{1} \leftrightarrow x_{2}, A \leftrightarrow B\right] .
\end{aligned}
$$

For $W^{+}$production in the $q g$ channel, we have

$$
\begin{aligned}
& \sum_{i, k \in Q \bar{Q}}\left(S^{i}\left(A, x_{1}\right) S^{g}\left(B, x_{2}\right) f_{q_{i}}^{N}\left(x_{1}, Q^{2}\right) f_{g}^{N}\left(x_{2}, Q^{2}\right)+\left[x_{1} \leftrightarrow x_{2}, A \leftrightarrow B\right]\right) C^{\mathrm{if}}\left(q_{i}, q_{k}\right) \\
& =B S^{g}\left(B, x_{2}\right) f_{g}^{p}\left(x_{2}, Q^{2}\right)\left[S^{u}\left(A, x_{1}\right)\left\{Z_{A} f_{u}^{p}\left(x_{1}, Q^{2}\right)+N_{A} f_{u}^{n}\left(x_{1}, Q^{2}\right)\right\}\right. \\
& \left.+S^{\bar{d}}\left(A, x_{1}\right)\left\{Z_{B} f_{\bar{d}}^{p}\left(x_{2}, Q^{2}\right)+N_{B} f_{\bar{d}}^{n}\left(x_{2}, Q^{2}\right)\right\}+A\left\{S^{\bar{s}}\left(A, x_{1}\right) f_{\bar{s}}^{p}\left(x_{1}, Q^{2}\right)+S^{c}\left(A, x_{1}\right) f_{c}^{p}\left(x_{1}, Q^{2}\right)\right\}\right] \\
& +\left[x_{1} \leftrightarrow x_{2}, A \leftrightarrow B\right] .
\end{aligned}
$$

Now the couplings do not enter explicitly for $W^{+}$and $W^{-}$production because each distribution is multiplied by $\left(\cos ^{2} \theta_{C}+\sin ^{2} \theta_{C}\right)$. Finally, the $q g$ convolution for $W^{-}$production is

$$
\begin{aligned}
& \sum_{i, k \in Q \bar{Q}}\left(S^{i}\left(A, x_{1}\right) S^{g}\left(B, x_{2}\right) f_{q_{i}}^{N}\left(x_{1}, Q^{2}\right) f_{g}^{N}\left(x_{2}, Q^{2}\right)+\left[x_{1} \leftrightarrow x_{2}, A \leftrightarrow B\right]\right) C^{\mathrm{if}}\left(q_{i}, q_{k}\right) \\
& =B S^{g}\left(B, x_{2}\right) f_{g}^{p}\left(x_{2}, Q^{2}\right)\left[S^{\bar{u}}\left(A, x_{1}\right)\left\{Z_{A} f_{\bar{u}}^{p}\left(x_{1}, Q^{2}\right)+N_{A} f_{\bar{u}}^{n}\left(x_{1}, Q^{2}\right)\right\}\right. \\
& \left.+S^{d}\left(A, x_{1}\right)\left\{Z_{B} f_{d}^{p}\left(x_{2}, Q^{2}\right)+N_{B} f_{d}^{n}\left(x_{2}, Q^{2}\right)\right\}+A\left\{S^{s}\left(A, x_{1}\right) f_{s}^{p}\left(x_{1}, Q^{2}\right)+S^{\bar{c}}\left(A, x_{1}\right) f_{\bar{c}}^{p}\left(x_{1}, Q^{2}\right)\right\}\right] \\
& +\left[x_{1} \leftrightarrow x_{2}, A \leftrightarrow B\right] .
\end{aligned}
$$




\section{REFERENCES}

[1] K.J. Eskola, K. Kajantie and J. Lindfors, Nucl. Phys. B323, 37 (1989).

[2] V. Emel'yanov, A. Khodinov, S.R. Klein and R. Vogt, Phys. Rev. C61, 044904 (2000).

[3] R. Vogt, Phys. Rept. 310, 197 (1999).

[4] H. Satz, Rept. Prog. Phys. 63, 1511 (2000).

[5] M.C. Abreu et al. (NA50 Collab.), Phys. Lett. B410 (1997) 327, 337.

[6] Z. Lin and R. Vogt, Nucl. Phys. B544, 339 (1999).

[7] J. Gunion and R. Vogt, Nucl. Phys. B492, 301 (1997).

[8] V. Kartvelishvili, R. Kvatadze and R. Shanidze, Phys. Lett. 356, 589 (1995).

[9] V. Emel'yanov, A. Khodinov, S.R. Klein and R. Vogt, Phys. Rev. Lett. 81, 1801 (1998).

[10] F. Abe et al., Phys. Rev. Lett. 81, 5754 (1998).

[11] C.W. deJager, H. deVries and C. deVries, Atomic Data and Nuclear Data Tables 14, 485 (1974).

[12] J.J. Aubert et al., Nucl. Phys. B293, 740 (1987); M. Arneodo, Phys. Rept. 240, 301 (1994).

[13] T. Kitagaki et al., Phys. Lett. 214, 281 (1988).

[14] K.J. Eskola, Z. Phys. C 51, 633 (1991).

[15] V. Emel'yanov, A. Khodinov, S.R. Klein, and R. Vogt, Phys. Rev. C56, 2726 (1997).

[16] V. Emel'yanov, A. Khodinov, S.R. Klein, and R. Vogt, Phys. Rev. C59, 1860 (1999).

[17] R. Hamberg, W.L. van Neerven and T. Matsuura, Nucl. Phys. B359, 343 (1991).

[18] B.W. Harris, J. Smith, and R. Vogt, Nucl. Phys. B461, 181 (1996).

[19] A.D. Martin, R.G. Roberts, and W.J. Stirling, and R.S. Thorne, Eur. Phys. J. C4, 463 (1998).

[20] K.J. Eskola, J. Qiu and J. Czyzewski, private communication.

[21] K.J. Eskola, Nucl. Phys. B400, 240 (1993).

[22] M. Glück, E. Reya, and A. Vogt, Z. Phys. C53, 127 (1992).

[23] K.J. Eskola, V.J. Kolhinen and P.V. Ruuskanen, Nucl. Phys. B535, 351 (1998).

[24] K.J. Eskola, V.J. Kolhinen and C.A. Salgado, Eur. Phys. J. C9, 61 (1999).

[25] A.H. Mueller and J.-W. Qiu, Nucl. Phys. B268, 427 (1986).

[26] L.V. Gribov, E.M. Levin and M.G. Ryskin, Phys. Rept. 100, 1 (1983).

[27] A.L. Ayala, M.B. Gay Ducati and E.M. Levin, Nucl. Phys. B493, 305 (1997).

[28] Z. Huang, H. Jung Lu and I. Sarcevic, Nucl. Phys. A637 79, (1998).

[29] L.D. Landau and I.Ya. Pomeranchuk, Dokl. Akad. Nauk SSSR 92 535, 735, (1953); A.B. Migdal, Phys. Rev. 103 1811, (1956); S.R. Klein, Rev. Mod. Phys. 71 1501, (1999).

[30] D. Kahana, in proceedings of RHIC Summer Study '96: Theory Workshop on Relativistic Heavy Ion Collisions, edited by D.E. Kahana and Y. Pang, BNL-52514, p. 175; Y. Pang, ibid, p. 193.

[31] S. Kumano and F.E. Close, Phys. Rev. C 41 1855, (1990).

[32] F.E. Close, R.G. Roberts and G.G. Ross, Phys. Lett. B142 202, (1984).

[33] P. Castorina and A. Donnachie, Z. Phys. C 49, 481 (1991).

[34] G.L. Li, K.F. Liu and G.E. Brown, Phys. Lett. B213, 531 (1988).

[35] H.L. Lai et al., Eur. Phys. J. C12, 375 (2000).

[36] J.I. Kapusta and S.M.H. Wong, Phys. Rev. D62, 037301 (2000).

[37] W. Geist, private communication. 


\section{TABLES}

\begin{tabular}{ccccc}
\hline \hline Detector & $\sigma(S=1)(\mathrm{nb})$ & $\sigma\left(S=S_{1}\right)(\mathrm{nb})$ & $\sigma\left(S=S_{2}\right)(\mathrm{nb})$ & $\sigma\left(S=S_{3}\right)(\mathrm{nb})$ \\
\hline \multirow{2}{*}{ CMS } & 16.10 & 11.37 & 11.22 & 14.92 \\
ALICE & 6.93 & 4.87 & 4.93 & 6.56 \\
& & $W^{+}$ & & \\
CMS & 25.18 & 17.39 & 17.08 & 23.23 \\
ALICE & 10.84 & 7.39 & 7.45 & 10.19 \\
& & $W^{-}$ & & 24.58 \\
CMS & 26.63 & 18.39 & 18.12 & 10.54 \\
ALICE & 11.21 & 7.64 & 7.73 & \\
\hline \hline
\end{tabular}

TABLE I. Vector boson production cross sections in units of nb per nucleon pair in $\mathrm{Pb}+\mathrm{Pb}$ collisions at $5.5 \mathrm{TeV} /$ nucleon calculated with the MRST HO parton densities. Full azimuthal coverage is assumed. The corresponding $p p$ cross sections at $14 \mathrm{TeV}$ are $\sigma^{Z^{0}}=35.44 \mathrm{nb}(\mathrm{CMS})$, $14.94 \mathrm{nb}$ (ALICE), $\sigma^{W^{+}}=60.50 \mathrm{nb}(\mathrm{CMS}), 24.76 \mathrm{nb}$ (ALICE) and $\sigma^{W^{-}}=52.95 \mathrm{nb}(\mathrm{CMS}), 22.88$ $\mathrm{nb}$ (ALICE).

\begin{tabular}{ccccc}
\hline \hline Detector & $N(S=1)$ & $N\left(S=S_{1}\right)$ & $N\left(S=S_{2}\right)$ & $N\left(S=S_{3}\right)$ \\
\hline & & $Z^{0}$ & & \\
CMS & $2.94 \times 10^{4}$ & $2.07 \times 10^{4}$ & $2.05 \times 10^{4}$ & $2.72 \times 10^{4}$ \\
ALICE & $1.26 \times 10^{4}$ & $8.88 \times 10^{3}$ & $8.99 \times 10^{3}$ & $1.20 \times 10^{4}$ \\
& & $W^{+}$ & \\
CMS & $4.59 \times 10^{4}$ & $3.17 \times 10^{4}$ & $3.12 \times 10^{4}$ & $4.24 \times 10^{4}$ \\
ALICE & $1.98 \times 10^{4}$ & $1.35 \times 10^{4}$ & $1.36 \times 10^{4}$ & $1.86 \times 10^{4}$ \\
& & $W^{-}$ & & \\
CMS & $4.86 \times 10^{4}$ & $3.35 \times 10^{4}$ & $3.31 \times 10^{4}$ & $4.48 \times 10^{4}$ \\
ALICE & $2.04 \times 10^{4}$ & $1.39 \times 10^{4}$ & $1.41 \times 10^{4}$ & $1.92 \times 10^{4}$ \\
\hline \hline
\end{tabular}

TABLE II. Number of vector bosons produced at $b=0$ in a one month $\left(10^{6} \mathrm{~s}\right) \mathrm{Pb}+\mathrm{Pb} \mathrm{LHC}$ run at $5.5 \mathrm{TeV} /$ nucleon. Note that no decay branching ratios have been included. 


\section{FIGURES}
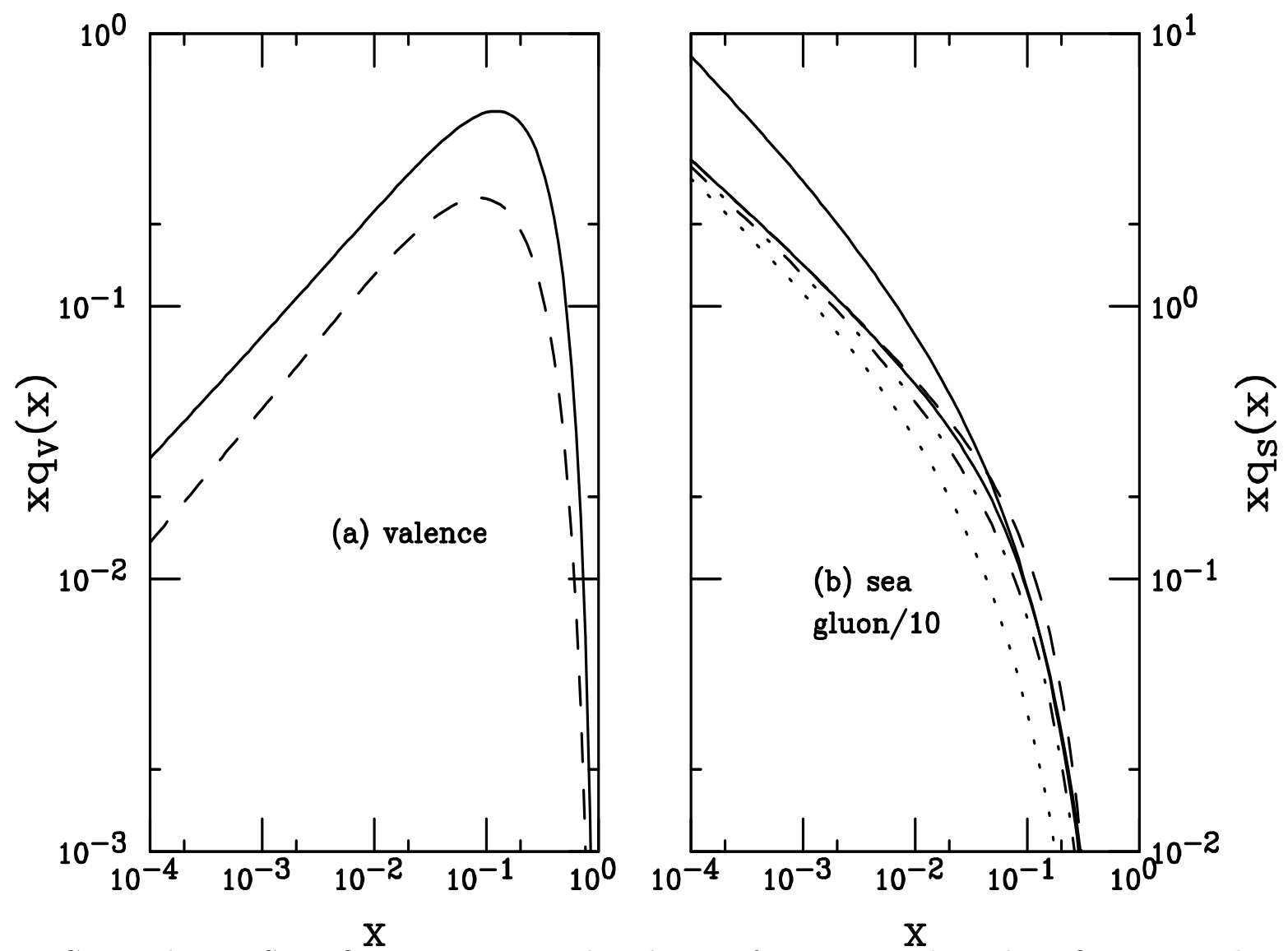

FIG. 1. The MRST HO proton parton distribution functions evaluated at $Q=m_{Z}$. The up (solid) and down (dashed) valence distributions are given in (a) while the up (lower solid), down (dashed), strange (dot-dashed) and charm (dotted) sea quark distributions are shown in (b), along with the gluon distribution (upper solid), reduced by a factor of 10 for comparison. 

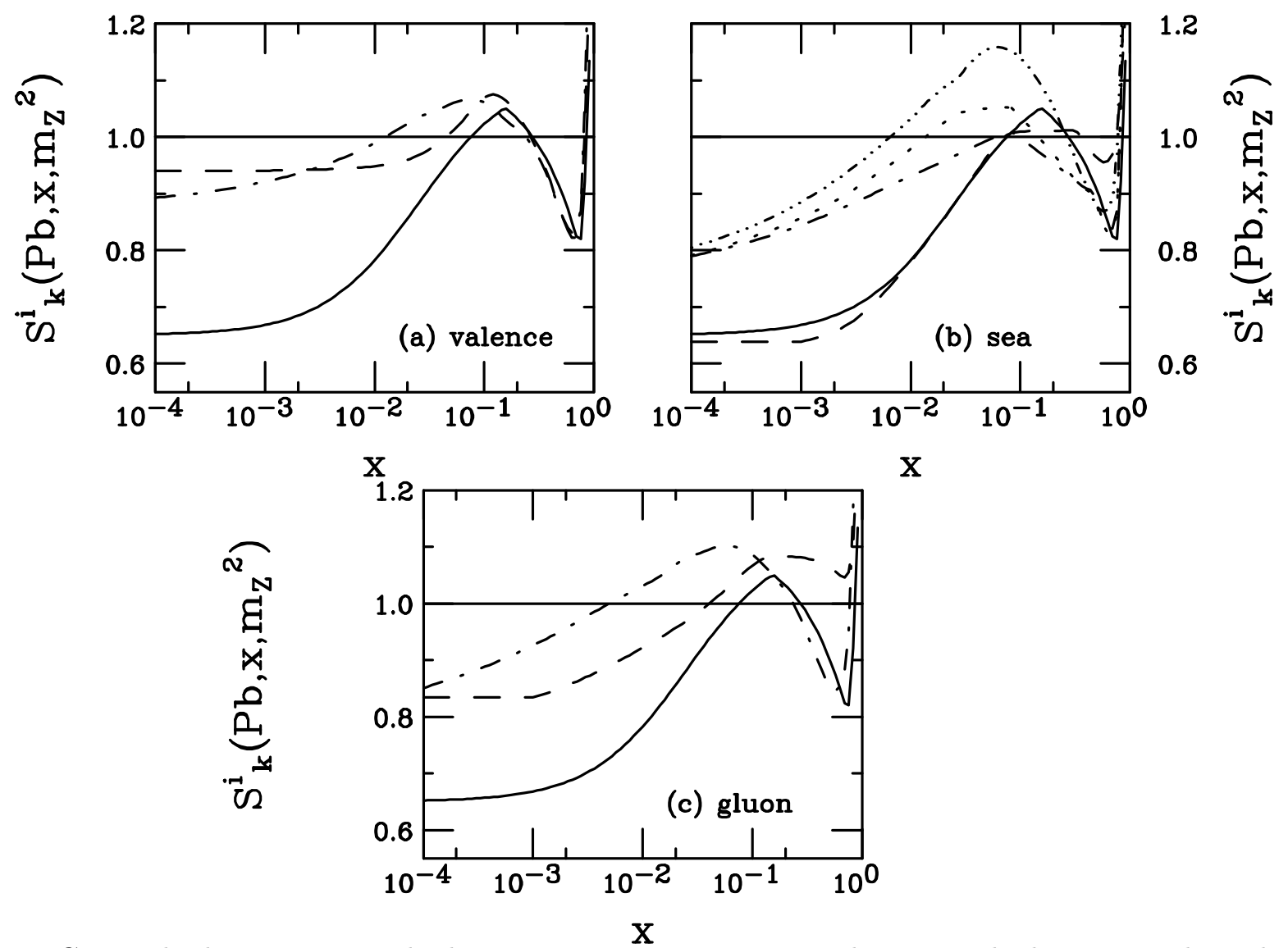

FIG. 2. The homogeneous shadowing parameterizations used in our calculations, evaluated at $Q^{2}=m_{Z}^{2}$. Valence shadowing is shown in (a) for the $S_{1}$ (solid), $S_{2}^{V}$ (dashed), and $S_{3}$ (dot-dashed) parameterizations. Sea quark shadowing is shown in (b) for $S_{1}$ (solid), $S_{2}^{S}$ (dashed), $S_{3}^{\bar{u}}=S_{3}^{\bar{d}}$ (dot-dashed), $S_{3}^{\bar{s}}$ (dotted) and $S_{3}^{\bar{c}}$ (dot-dot-dot-dashed). Gluon shadowing is shown in (c) for $S_{1}$ (solid), $S_{2}^{g}$ (dashed) and $S_{3}^{g}$ (dot-dashed). 


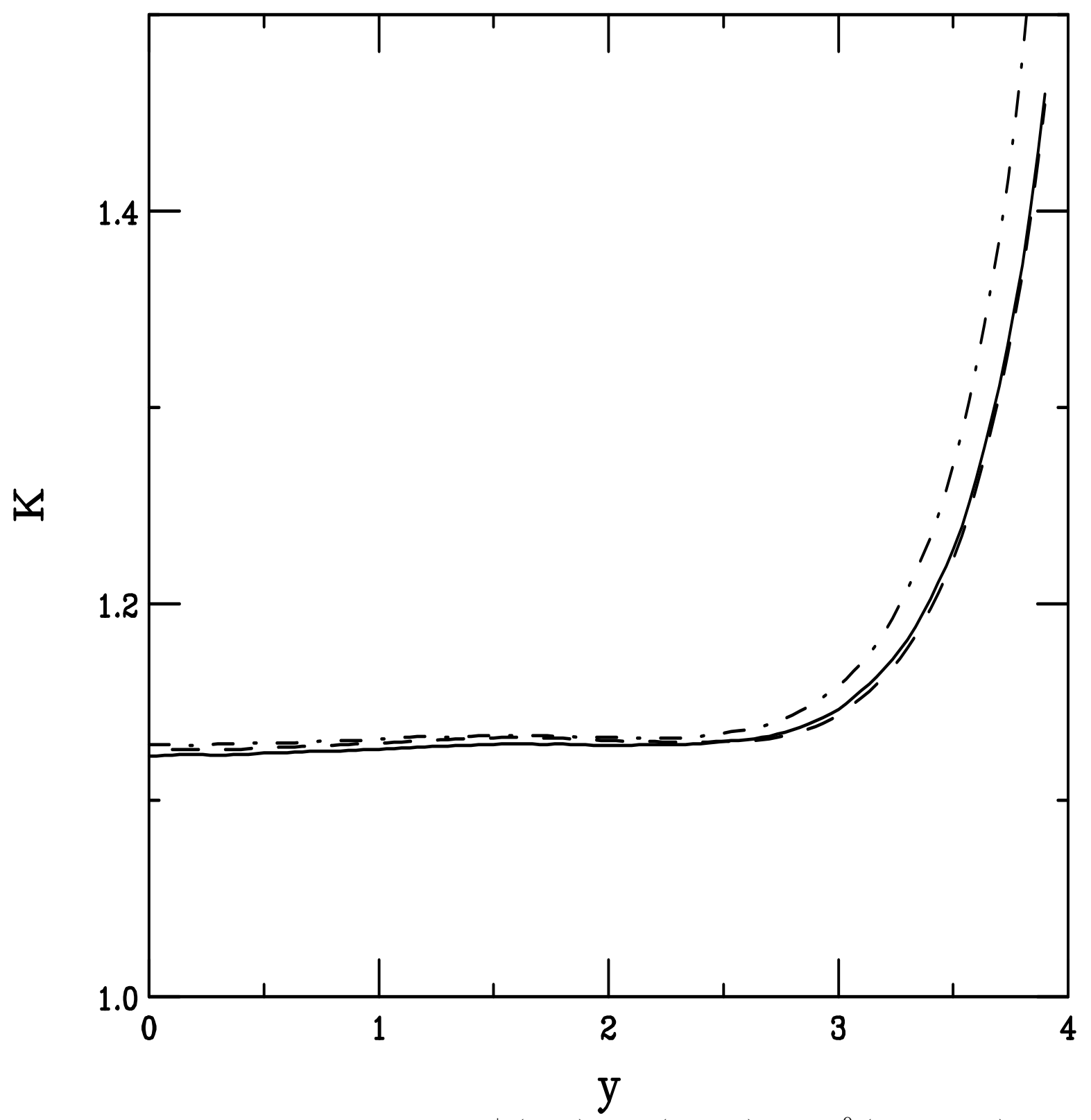

FIG. 3. The $K$ factors with $S=1$ for $W^{+}$(solid), $W^{-}$(dashed), and $Z^{0}$ (dot-dashed) production are shown. 


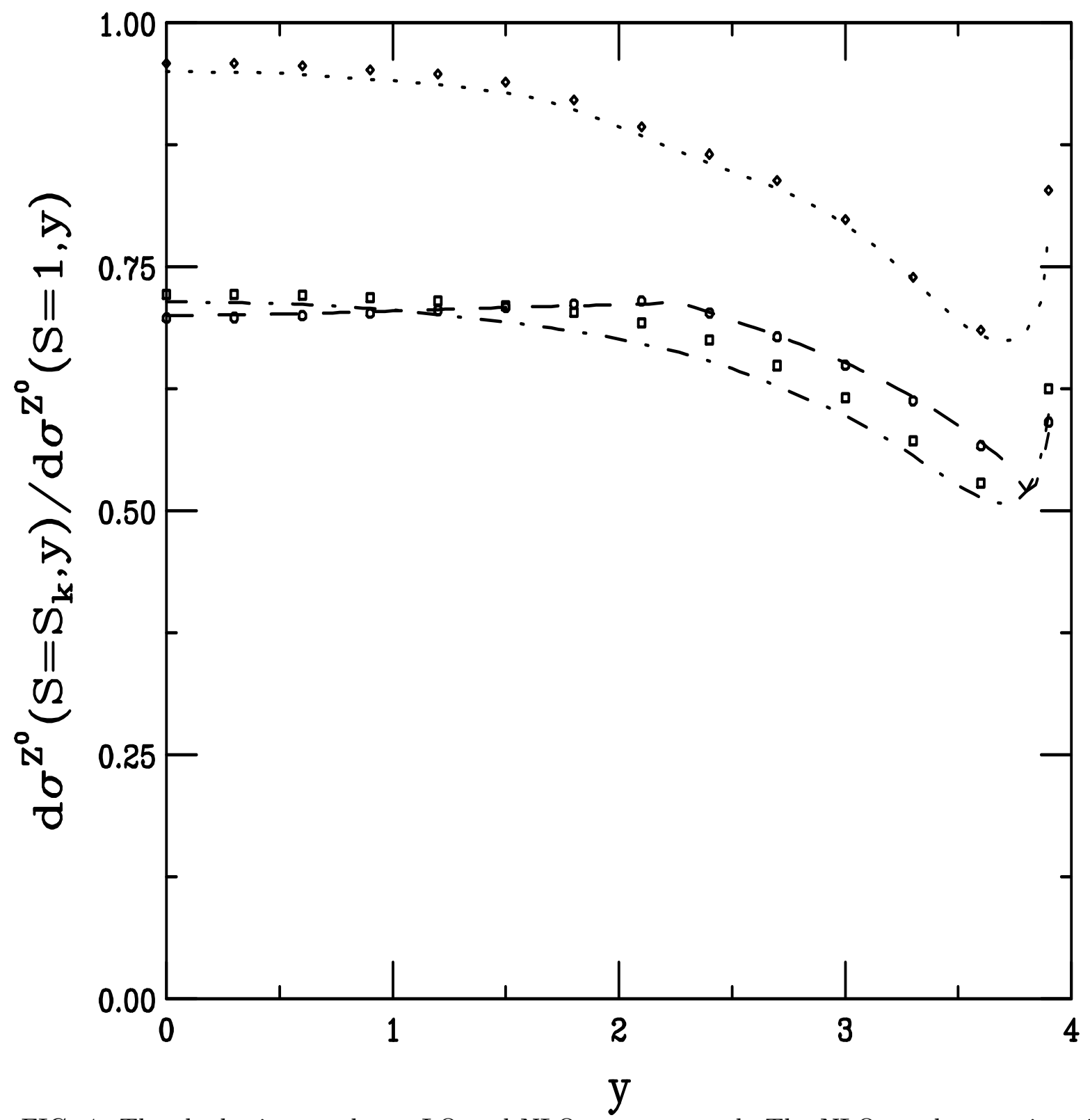

FIG. 4. The shadowing results at LO and NLO are compared. The NLO results are given in the dashed, $S_{1}$, dot-dashed, $S_{2}$, and dotted, $S_{3}$, lines. The LO shadowing ratios for $S_{1}$, circles, $S_{2}$, squares, and $S_{3}$, diamonds, are also shown. 


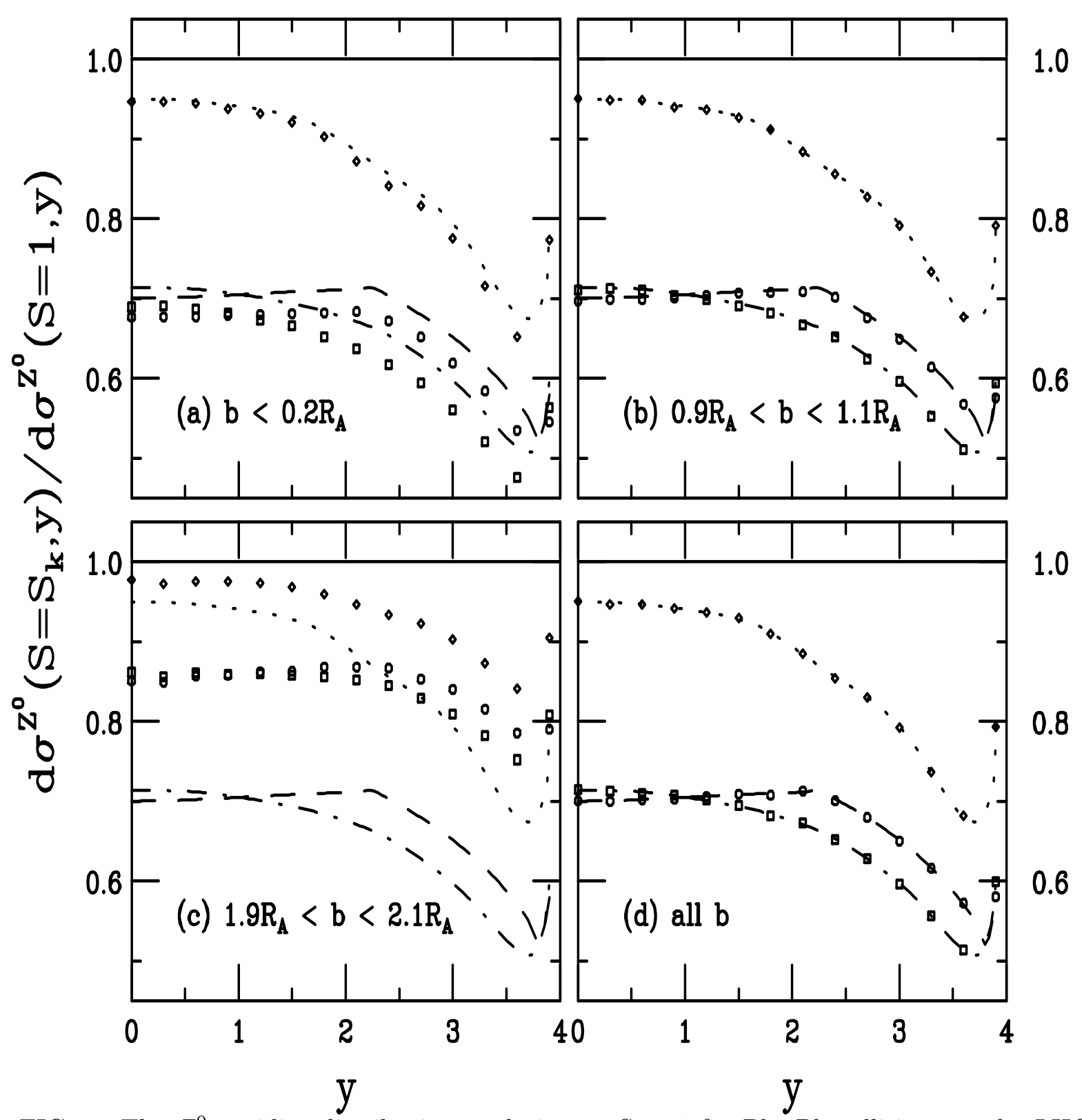

FIG. 5. The $Z^{0}$ rapidity distributions, relative to $S=1$ for $\mathrm{Pb}+\mathrm{Pb}$ collisions at the LHC, calculated with the MRST HO distributions. Central, $b<0.2 R_{A}$, semi-central, $0.9 R_{A}<b<1.1 R_{A}$, and peripheral, $1.9 R_{A}<b<2.1 R_{A}$ impact parameters are shown along with the integral over all $b$. The homogeneous shadowing results are given in the dashed, $S_{1}$, dot-dashed, $S_{2}$, and dotted, $S_{3}$, lines. The inhomogeneous shadowing ratios for $S_{1 \mathrm{ws}}$, circles, $S_{2}$ ws, squares, and $S_{3}$ ws, diamonds, are also shown. 


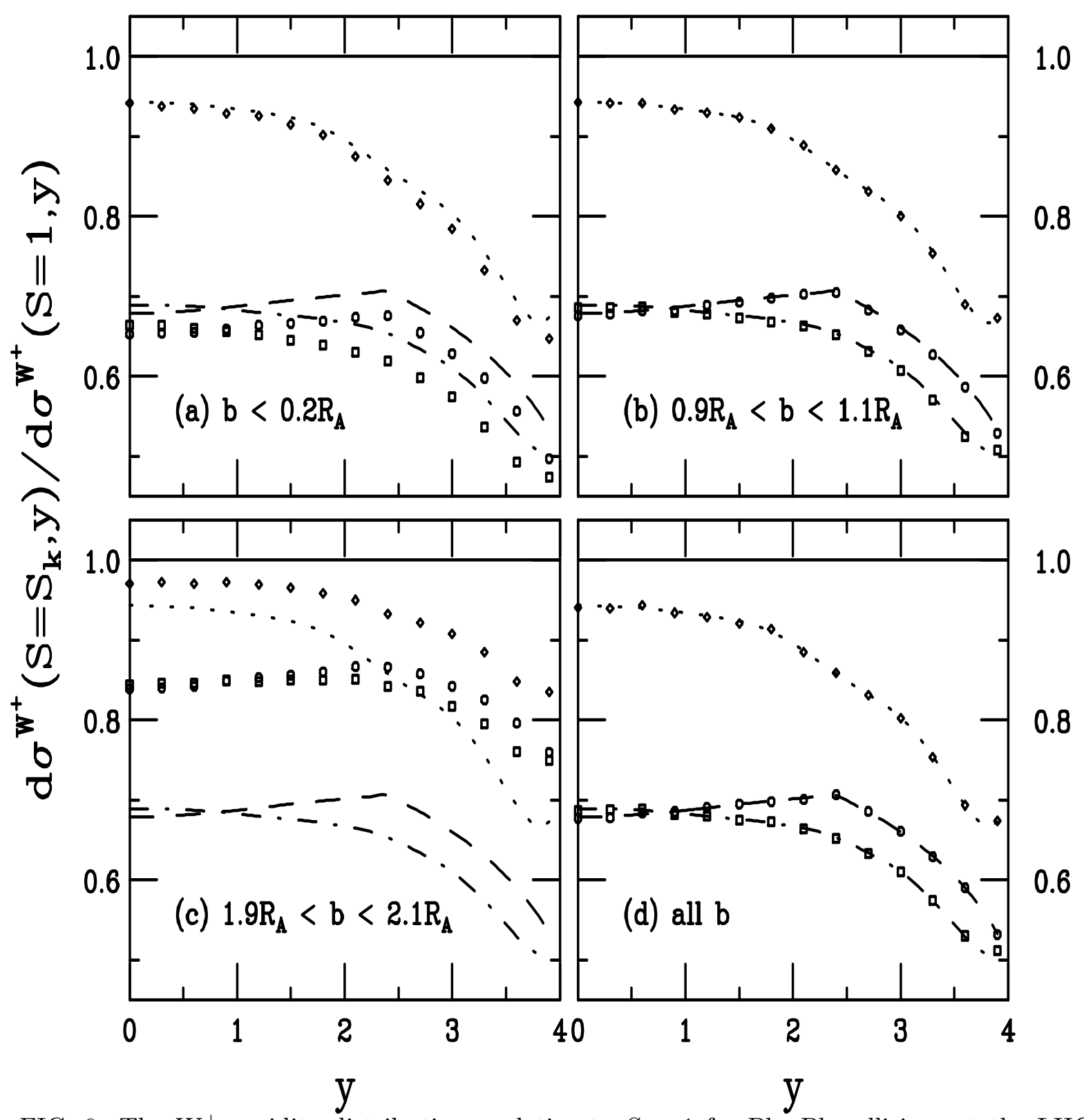

FIG. 6. The $W^{+}$rapidity distributions, relative to $S=1$ for $\mathrm{Pb}+\mathrm{Pb}$ collisions at the LHC, calculated with the MRST HO distributions. Central, $b<0.2 R_{A}$, semi-central, $0.9 R_{A}<b<1.1 R_{A}$, and peripheral, $1.9 R_{A}<b<2.1 R_{A}$ impact parameters are shown along with the integral over all $b$. The homogeneous shadowing results are given in the dashed, $S_{1}$, dot-dashed, $S_{2}$, and dotted, $S_{3}$, lines. The inhomogeneous shadowing ratios for $S_{1 \mathrm{ws}}$, circles, $S_{2}$ Ws, squares, and $S_{3}$ ws, diamonds, are also shown. 


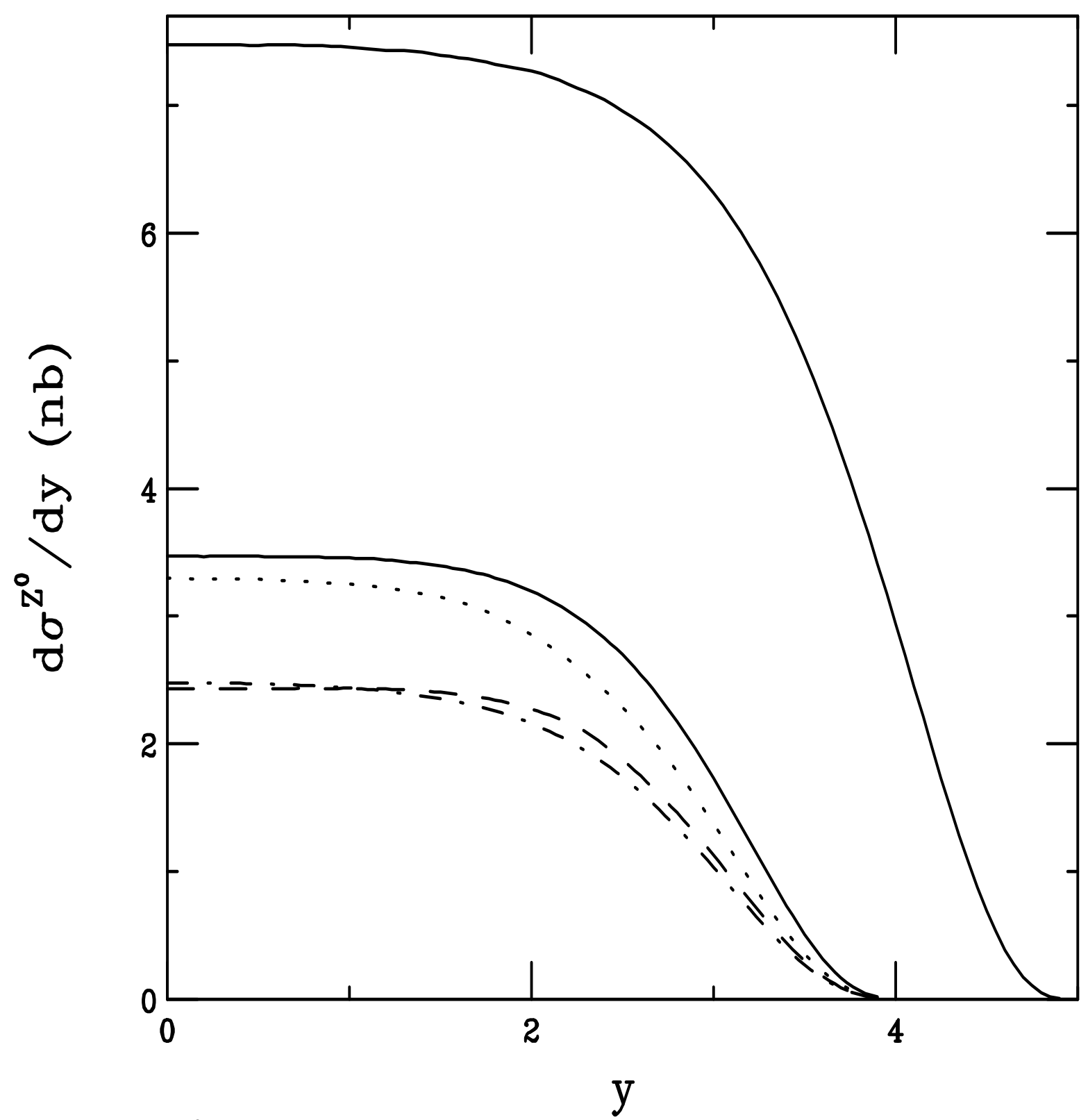

FIG. 7. The $Z^{0}$ rapidity distributions in $p p$ and $\mathrm{Pb}+\mathrm{Pb}$ collisions, calculated with the MRST HO distributions. The upper solid curve is the $p p$ result at $14 \mathrm{TeV}$ while the lower solid curve is the $\mathrm{Pb}+\mathrm{Pb}$ distribution at $5.5 \mathrm{TeV} /$ nucleon pair with no shadowing. The homogeneous shadowing results for $\mathrm{Pb}+\mathrm{Pb}$ collisions are given in the dashed, $S_{1}$, dot-dashed, $S_{2}$, and dotted, $S_{3}$, lines. 


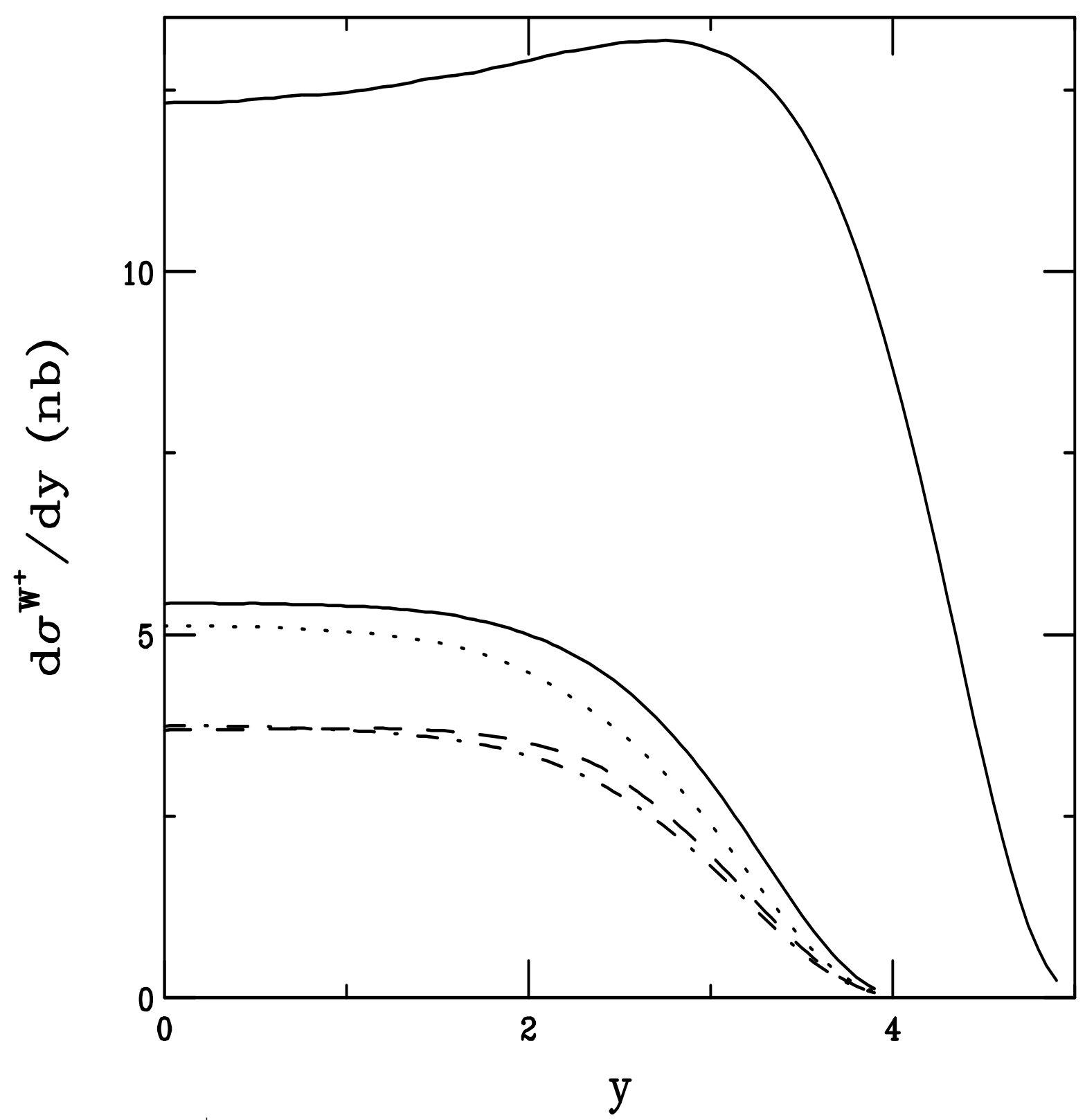

FIG. 8. The $W^{+}$rapidity distributions in $p p$ and $\mathrm{Pb}+\mathrm{Pb}$ collisions, calculated with the MRST HO distributions. The upper solid curve is the $p p$ result at $14 \mathrm{TeV}$ while the lower solid curve is the $\mathrm{Pb}+\mathrm{Pb}$ distribution at $5.5 \mathrm{TeV} /$ nucleon pair with no shadowing. The homogeneous shadowing results for $\mathrm{Pb}+\mathrm{Pb}$ collisions are given in the dashed, $S_{1}$, dot-dashed, $S_{2}$, and dotted, $S_{3}$, lines. 


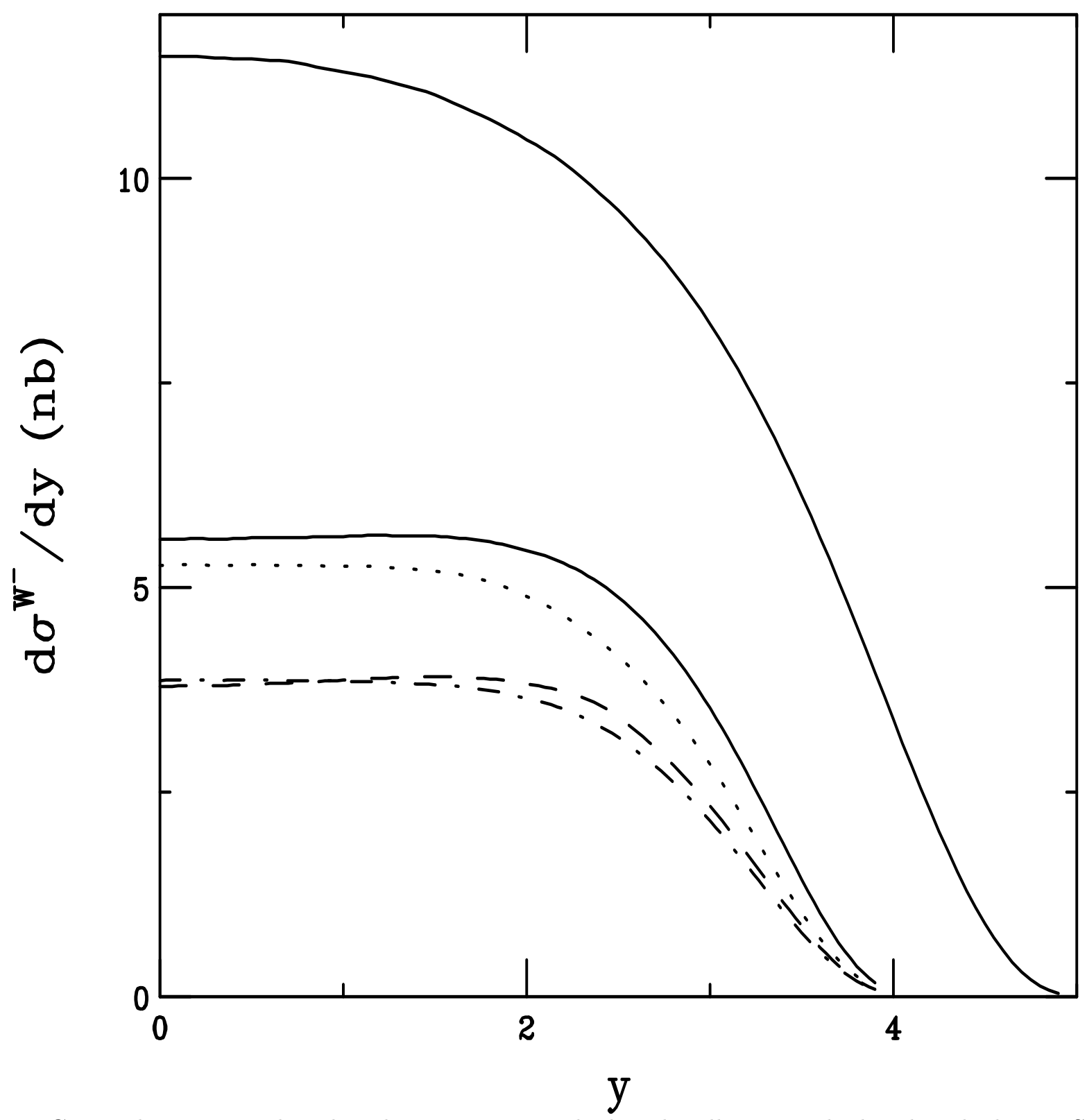

FIG. 9. The $W^{-}$rapidity distributions in $p p$ and $\mathrm{Pb}+\mathrm{Pb}$ collisions, calculated with the MRST HO distributions. The upper solid curve is the $p p$ result at $14 \mathrm{TeV}$ while the lower solid curve is the $\mathrm{Pb}+\mathrm{Pb}$ distribution at $5.5 \mathrm{TeV} /$ nucleon pair with no shadowing. The homogeneous shadowing results for $\mathrm{Pb}+\mathrm{Pb}$ collisions are given in the dashed, $S_{1}$, dot-dashed, $S_{2}$, and dotted, $S_{3}$, lines. 


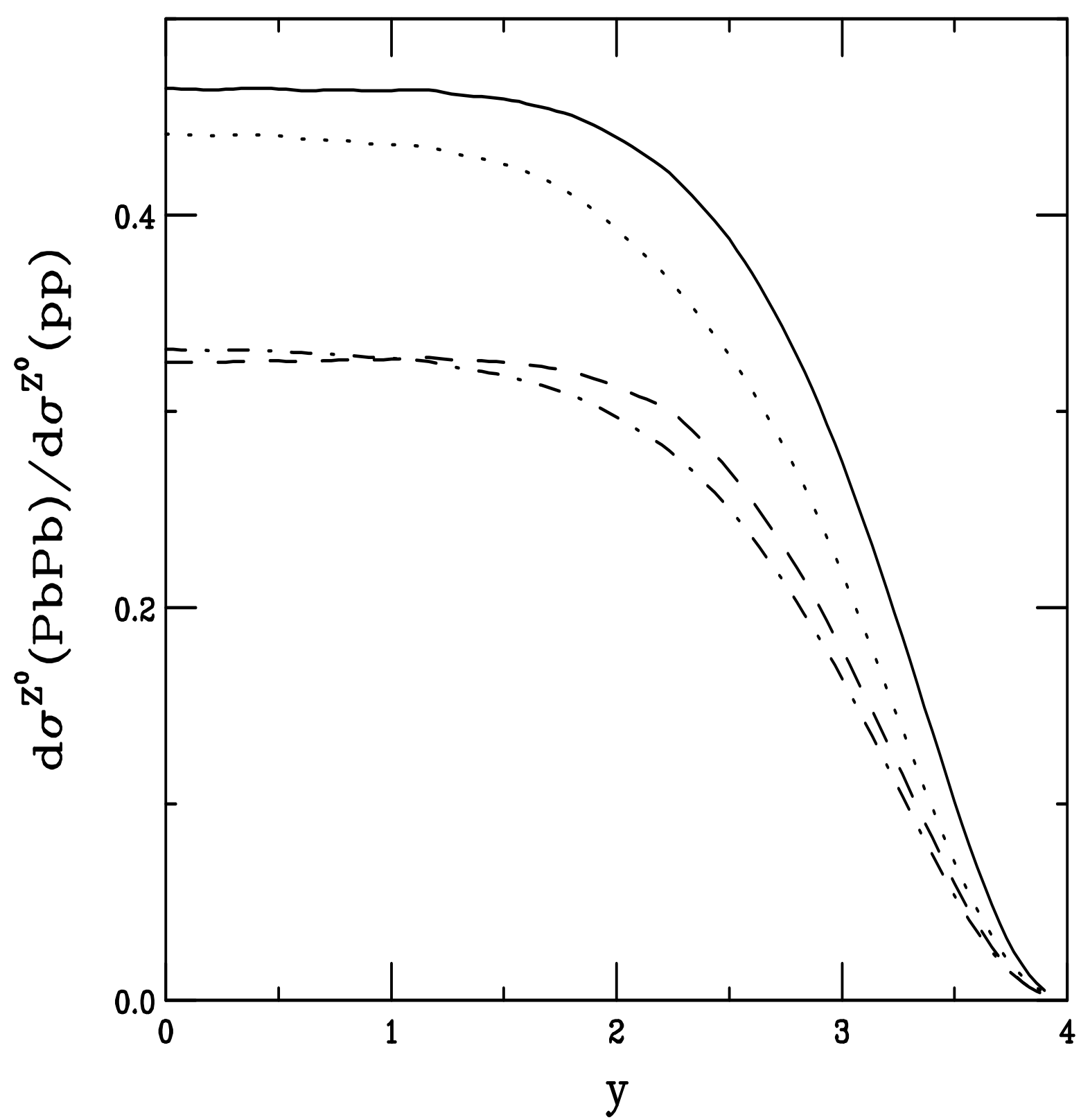

FIG. 10. The ratios of the $Z^{0}$ rapidity distributions in $\mathrm{Pb}+\mathrm{Pb}$ collisions relative to $p p$ collisions, calculated with the MRST HO distributions. The solid curve is the ratio without shadowing. The homogeneous shadowing results are given in the dashed, $S_{1}$, dot-dashed, $S_{2}$, and dotted, $S_{3}$, lines. 


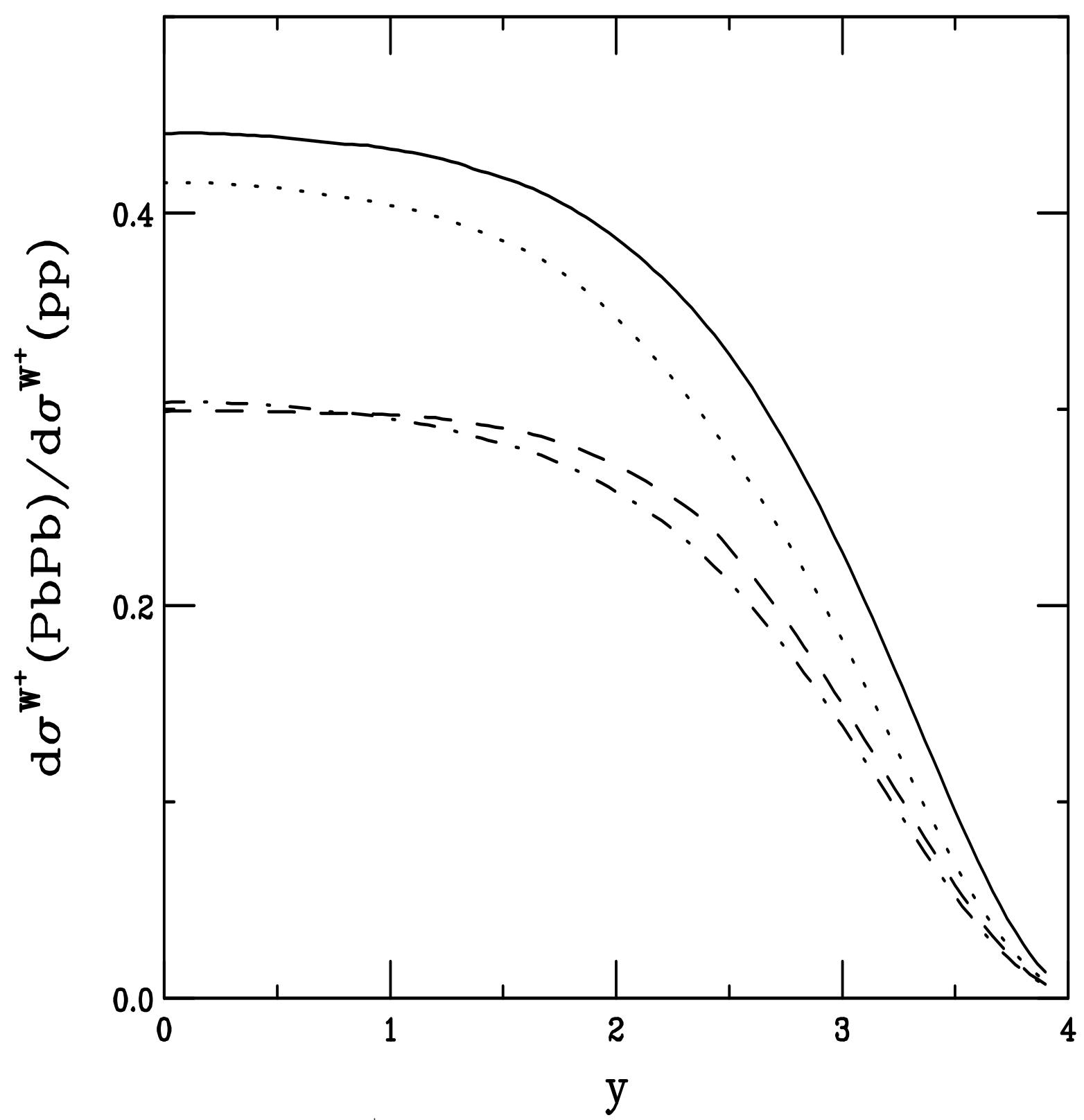

FIG. 11. The ratios of the $W^{+}$rapidity distributions in $\mathrm{Pb}+\mathrm{Pb}$ collisions relative to $p p$ collisions, calculated with the MRST HO distributions. The solid curve is the ratio without shadowing. The homogeneous shadowing results are given in the dashed, $S_{1}$, dot-dashed, $S_{2}$, and dotted, $S_{3}$, lines. 


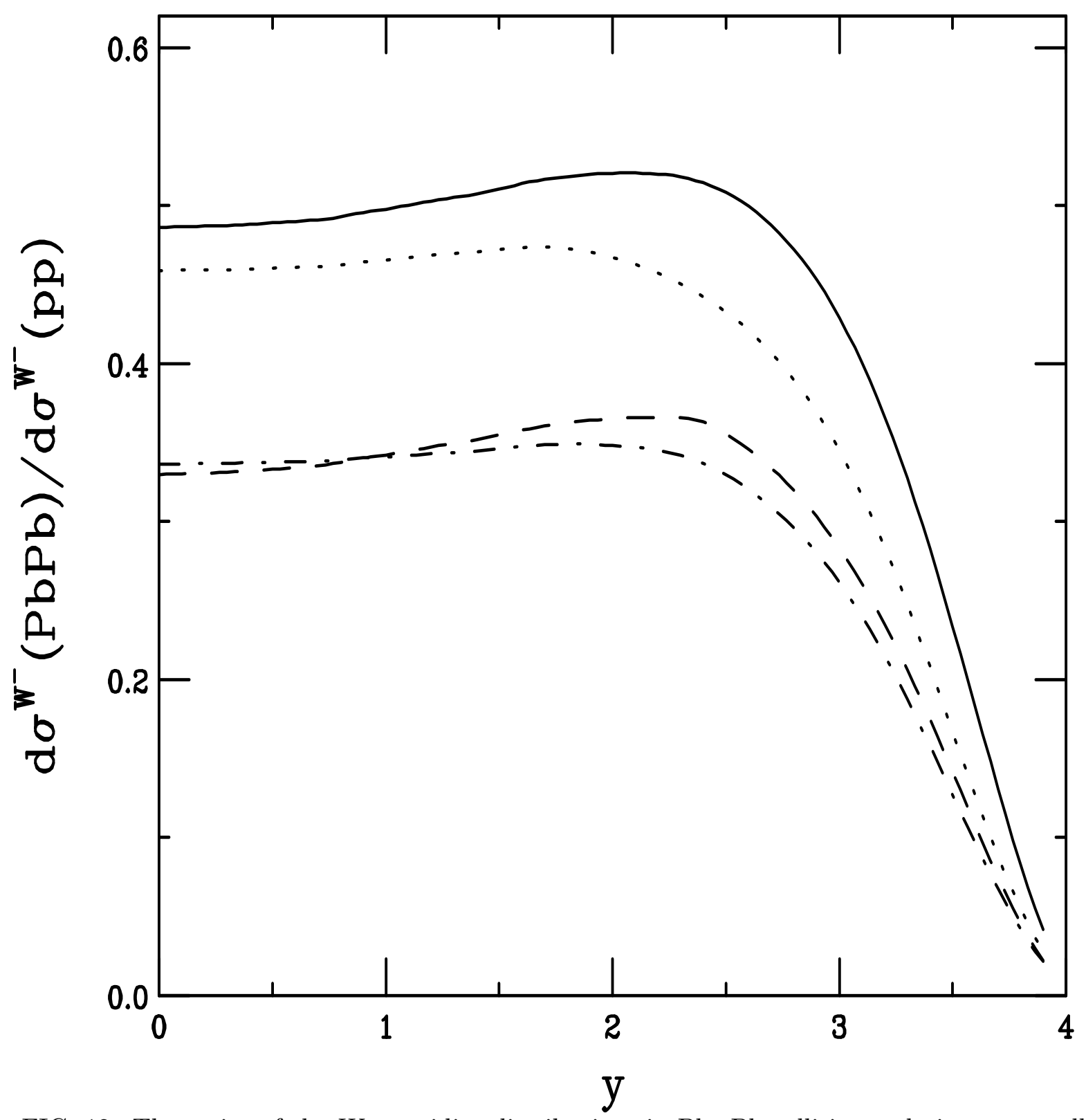

FIG. 12. The ratios of the $W^{-}$rapidity distributions in $\mathrm{Pb}+\mathrm{Pb}$ collisions relative to $p p$ collisions, calculated with the MRST HO distributions. The solid curve is the ratio without shadowing. The homogeneous shadowing results are given in the dashed, $S_{1}$, dot-dashed, $S_{2}$, and dotted, $S_{3}$, lines. 